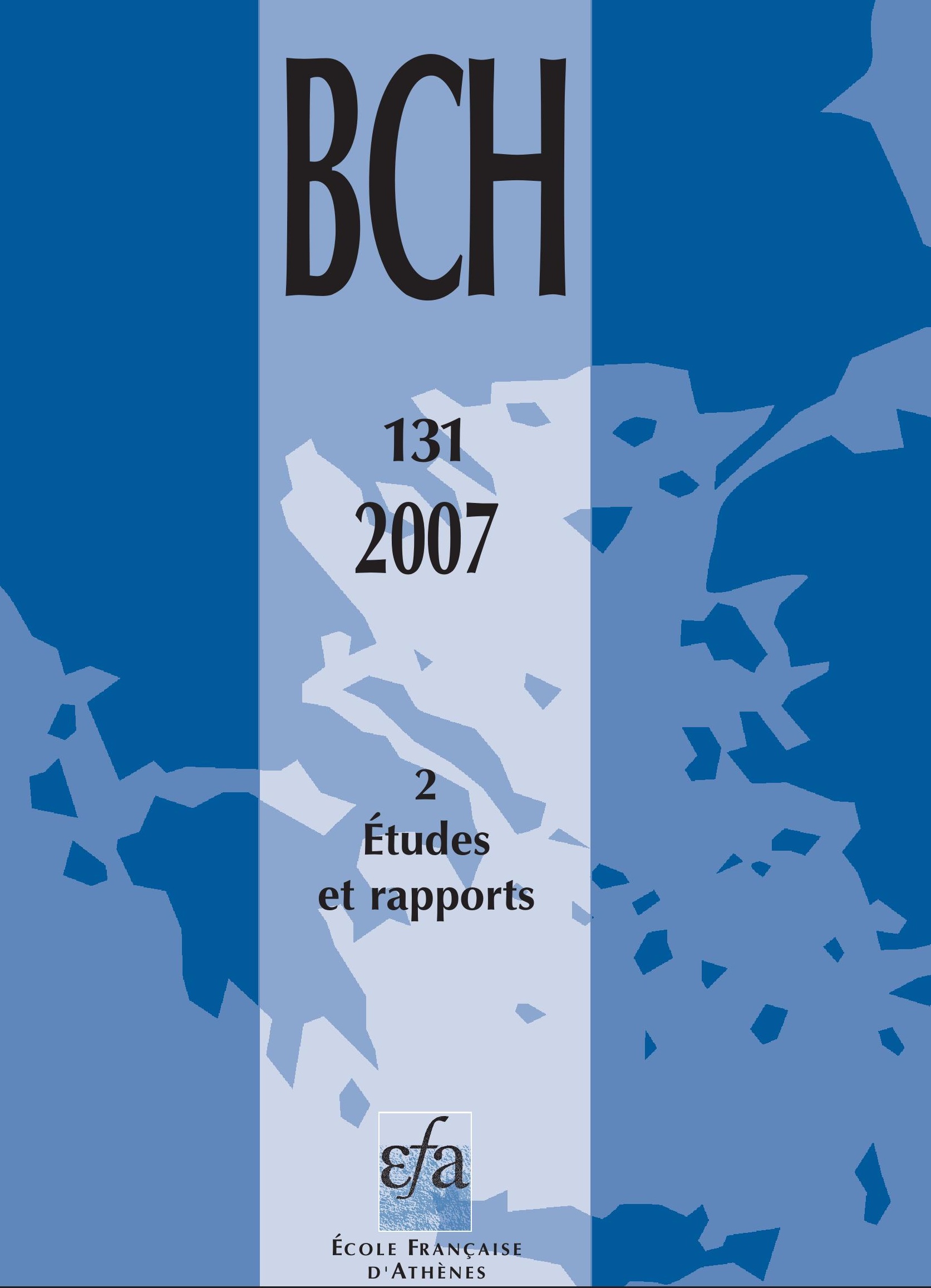

BULLETIN DE CORRESPONDANCE HELLÉNIQUE 


\section{AVIS AUX LECTEURS}

Partageant une longue tradition, l'École française d'Athènes et la British School at Athens diffusent auprès de la communauté scientifique le résultat de l'activité archéologique conduite en Grèce et dans certaines régions du monde hellénique. Depuis 1920, l'École française d'Athènes consacre une partie du Bulletin de Correspondance hellénique à la chronique des travaux archéologiques réalisés en Grèce, à Chypre et, selon un rythme bisannuel, dans le Bosphore Cimmérien. De son côté, la British School at Athens compile un bilan annuel similaire, Archaeology in Greece, publié en association avec la Society for the Promotion of Hellenic Studies comme partie constitutive des Archaeological Reports depuis 1955. Chacune des deux institutions avait un double défi à relever : faire face à une documentation croissante, d'une part ; utiliser des outils plus performants pour mieux faire circuler l'information scientifique et en permettre une meilleure utilisation, d'autre part. - L'École britannique a accepté sans hésitation le projet d'un programme commun que lui a proposé l'École française d'Athènes et les deux institutions ont décidé d'unir leurs efforts, pour proposer à partir de la fin de l'année 2009 une Chronique des fouilles en ligne consultable sur http://chronique.efa.gr.

Outre les articles relatifs à des opérations de terrain ou relevant de l'archéométrie, le second fascicule du $B C H$ ne comprendra donc plus désormais que les " Rapports sur les travaux de l'École française d'Athènes " proposés par les responsables de missions ou de programmes.

\section{AVIS AUX AUTEURS}

Depuis la parution du $B C H 130$ (2006), les tirages à part sont fournis aux auteurs sous format électronique et sont uniquement destinés à une utilisation privée. L'École française d'Athènes conserve le copyright sur les articles, qui ne peuvent donc être mis en accès libre sur quelque base de données ou par quelque portail que ce soit. - L'ensemble de la livraison sera disponible sur le portail Persée trois ans après sa parution (www.persee.fr). 


\section{SOMMAIRE DE LA LIVRAISON}

\section{I. Études}

Rozalia CHRISTIDOU,

Aperçu des industries osseuses de l'habitat protohistorique lacustre de Sovjan (bassin de Korçë, Albanie sud-orientale) 755-803

Éric FOUACHE, Claude COSANDEY, Lucie CEZ, Kosmas PAVLOPOULOS et Josette RENARD

Contexte géomorphologique du bassin de Sparte et ressources en eau du site néolithique de Kouphovouno (Péloponnèse, Grèce) 805-819

Maia POMADÈRE et Julien ZURBACH

Journées maliotes

Malia, ville et territoire : organisation des espaces et exploitation des ressources, colloque organisé à l'École française d'Athènes les 2-3 novembre 2007 821-887

Hélène WURMSER, Alexandre FARNOUX, Lionel FADIN et Stavroula APOSTOLAKOU

Missions topographiques à Latô I (2005-2007) : analyse critique du plan de J. Demargne et V. Seyk (1901) 889-924

\section{Rapports}

\section{RAPPORT SUR LES TRAVAUX DE L'ÉCOLE FRANÇAISE D'ATHÈNES EN 2006}

LES ACTIVITÉS DE L'EFA : ÉTUDES ET FORMATION À LA RECHERCHE

par Dominique MULLIEZ

Grèce

PHILIPPES d'après Samuel PROVOST 934-937

Thasos par Jean-Yves MARC, Pierre MOUGIN, Manuela WURCH-KOZELJ et Bernard HOLTZMANN. $938-947$

ARGOS par Anna PHILIPPA-TOUCHAIS, Gilles TOUCHAIS et d'après Anne PARIENTE et Christos PITÉROS. $948-971$

Kouphovouno (LACONIE) par William CAVANAGH, Christopher MEE et Josette RENARD $972-980$ 
DÉlos par Cécile DURVYE, Roland ÉTIENNE, Lionel FADIN, Myriam FINCKER, Jean-Charles MORETTI, Véronique PICARD et Hélène SIARD …_________. …982-1025

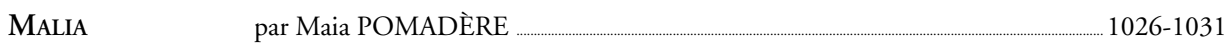

Chypre

AMATHONTE par P. AUPERT, Claire BALANDIER, Béatrice BLANDIN, Tony KOZELJ,

Pierre LERICHE, Thierry PETIT et Isabelle TASSIGNON …__ _ 1032-1049

Albanie

SOVJAN

par Pétrika LERA et Gilles TOUCHAIS

1050-1075

APOLLONIA par Pierre CABANES, Jean-Luc LAMBOLEY et Bashkim VREKAJ

1076-1097

BILLYS

par Nicolas BEAUDRY, Amélie BERTHON, Eduart BITRI,

Pascale CHEVALIER, Elio HOBDARI, Agron ISLAMI, Tony KOZELJ,

Skënder MUÇAJ, Etleva NALLBANI, Manon SAVARD

et Manuela WURCH-KOZELJ

$1098-1113$

DURRËS

par Arthur MULLER et Fatos TARTARI

$1114-1118$ 


\title{
Missions topographiques à Latô I (2005-2007) : analyse critique du plan de J. Demargne et V. Seyk (1901)
}

\author{
Hélène Wurmser, Alexandre Farnoux, Lionel Fadin, Stavroula Apostolakou
}

RÉSUMÉ Entre les années 2005 et 2007, le site de Latô en Crète a fait l'objet de trois nouvelles campagnes d'études topographiques et géomorphologiques. Les travaux menés sur l'ensemble du site ont notamment permis d'analyser le seul plan complet de la ville dont nous disposons encore aujourd'hui et qui fut l'œuvre de J. Demargne et de l'architecte V. Seyk en 1900. L'objectif était d'en établir l'intérêt documentaire et les limites d'utilisation, pour mieux préparer la production de nouveaux relevés et orienter la reprise des études sur le terrain. Pour ce faire, il nous a fallu tout d'abord retracer l'historique des documents graphiques antérieurs (I), puis analyser les travaux de J. Demargne et de V. Seyk (II), afin d'en mesurer la valeur à l'aune des recherches actuelles (III) et d'en établir l'intérêt pour la gestion patrimoniale du site (IV). Un deuxième article à paraître dans le $\mathrm{BCH}$, développera, à partir d'une documentation nouvelle, l'analyse urbaine et morphologique des vestiges de Latô.

ПЕРІ $\Lambda$ АН АПО

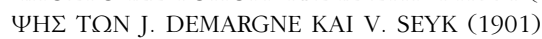

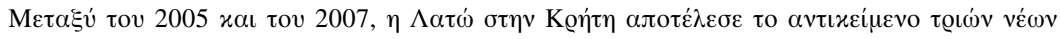

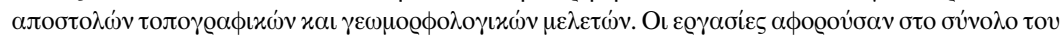

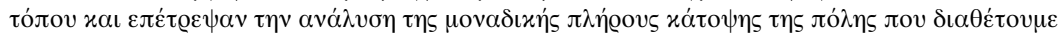

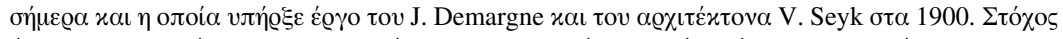

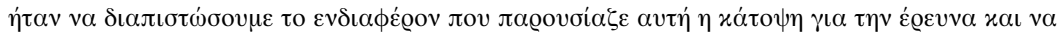

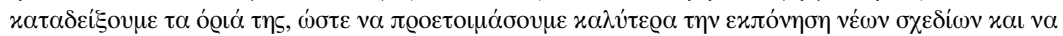

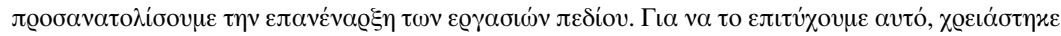

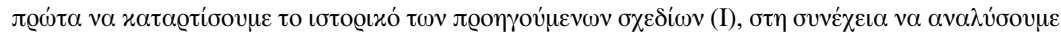

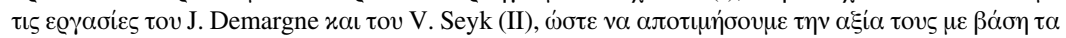

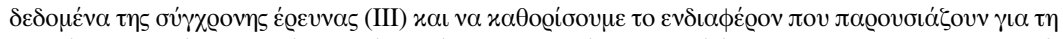

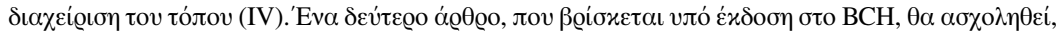

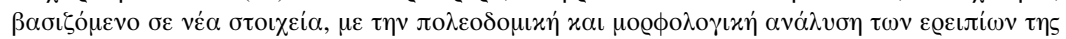

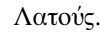

ABSTRACT TOPOGRAPHICAL MISSIONS TO LATO I (2005-2007): A CRITICAL ANALYSIS OF THE PLAN BY J. DEMARGNE AND V. SEYK (1901)

In the years 2005 and 2007 the site of Lato in Crete was the object of three new topographical and geomorphological study campaigns. The works carried out over the entire site permitted in particular the analysis of the only to date available complete plan of the city, that of J. Demargne and the architect V. Seyk undertaken in 1900 . The objectif was to establish its documentary interest and limits in use, and thus better prepare new measurements and guide renewed field work. To do this, it was first necessary (I) to retrace the history of previous graphic documentation, (II) to analyse the work of J. Demargne and V. Seyk, so as (III) to gauge its value against the standards of current research, and (IV) to establish its interest in terms of the cultural resource management of the site. A second paper to appear in $\mathrm{BCH}$ will develop, on basis of a new documentation, the urban and morphological analysis of the remains of Lato. 
Les travaux d'aménagement réalisés par la XXIV éphorie des Antiquités préhistoriques et classiques d'Aghios Nikolaos à Latô depuis 1999, ainsi que le développement des études régionales à l'Est et à l'Ouest du Mirambello ont favorisé en 2005 la reprise des travaux de l'École française d'Athènes dans la région. Trois campagnes de terrain ont été réalisées à Latô en 2005, 2006 et $2007^{1}$ pour évaluer la documentation existante et établir une nouvelle série de documents topographiques répondant à la fois aux exigences de la recherche et de la gestion des vestiges. Il a paru souhaitable d'inscrire ce travail dans un programme à l'échelle de la région du Mirambello, où l'École française d'Athènes avait mené des opérations de fouilles au début du XXe siècle, en exploitant les possibilités offertes par la constitution d'une base de données de type $\mathrm{SIG}^{2}$. Nous présentons ici les résultats des trois campagnes qui ont porté sur l'évaluation du plan de Latô réalisé parV. Seyk dans l'été 1900, lors de la fouille de J. Demargne. Ce plan constitue aujourd'hui encore le seul document de référence, pour le spécialiste comme pour le visiteur occasionnel. L'objectif était d'en établir l'intérêt documentaire et les limites d'utilisation, pour mieux préparer la production de nouveaux relevés et orienter la reprise des études sur le terrain. Pour ce faire, il nous a fallu tout d'abord retracer l'historique des document graphiques antérieurs (I), puis analyser les travaux de J. Demargne et de V. Seyk (II), afin d'en mesurer la valeur à l'aune des recherches actuelles (III) et d'en établir l'intérêt pour la gestion patrimoniale du site (IV). Un deuxième article développera, à partir d'une documentation nouvelle, l'analyse urbaine et morphologique des vestiges de Latô.

* H. Wurmser, membre de $4{ }^{\mathrm{e}}$ année de l'École française d'Athènes ; A. Farnoux, professeur d'histoire de l'art et d'archéologie à l'université Paris IV-Sorbonne; L. Fadin, ingénieur topographe à l'École française d'Athènes; S. Apostolakou, éphore de la XXIVe éphorie des antiquités préhistoriques et classiques d'Aghios Nikolaos (Crète)

1. Effectuées en été ou au printemps sur le terrain, les missions ont été complétées par une campagne d'étude à Athènes en février 2007 et par une campagne de compléments et de vérification la même année. Elles sont le fruit d'une collaboration entre L'EFA et la XXIVe éphorie des Antiquités préhistoriques et classiques, avec la participation de l'université Paris IV-Sorbonne et le soutien de l'école doctorale VI d'Histoire de l'Art et d'Archéologie.

2. Le projet s'inscrit dans le programme quadriennal 2008-2011 de l'EFA. M. Ghilardi, géographe, est chargé plus particulièrement de la réalisation du SIG et de l'analyse géomorphologique de la région. 


\section{LES DOCUMENTS GRAPHIQUES ANTÉRIEURS AU PLAN DEMARGNE - SEYK (A. F., H. W.)}

Lorsque J. Demargne entreprend des fouilles au lieu-dit Goulas en avril 1899, il n'est pas le premier à s'être intéressé aux ruines de cette région de la Crète. Le massif du Mirambello avait déjà suscitél'intérêt des voyageurs comme des premiers archéologues quelques années auparavant.

Les rares voyageurs qui visitent Goulas au courant du XIXe siècle relèvent la présence de vestiges importants, relativement bien conservés. Rapidement relayés par les archéologues, ils décrivent un site caractérisé par des murs puissants et une situation géographique exceptionnelle. Les visites plus longues et les descriptions plus circonstanciées de F. Halbherr, L. Mariani et A. Evans conduisent, dans un deuxième temps, à l'identification du site de Goulas avec la ville ancienne de Latô, et à sa datation à l'époque mycénienne. Chaque étude est accompagnée de documents différents, croquis, relevés, plans, photographies, qui nourrissent la connaissance du site et fondent l'analyse archéologique. Dans la plupart des cas, l'ensemble de ces documents présente une double perspective, géographique et topographique.

\section{A) LES VOYAGEURS À PALAIOKASTRO/GOULAS}

Le premier témoignage que nous possédons sur l'existence de ruines au Nord du village de Kritsa est celui d'A. Fabreguettes, consul de France à La Canée. Au début de l'été 1834, il effectue un voyage en Crète orientale dont le compte rendu est présenté à la Société française de Géographie. À propos de sa visite dans la région de Kritsa, il écrit qu’à un mile de Kritsa se trouve Palaiokastro que les Grecs appellent aujourd'hui Aghios Antonios et qui doit être l'acropole de Lictium. Il précise que Tournefort ne l'a pas visité alors qu'il a vu Kritsa, et que Savary n' est jamais venu dans ce secteur. Les ruines sont d'un type ancien d'architecture militaire. Plusieurs styles sont reconnaissables parmi elles, depuis le pur cyclopéen jusqu'à l'hellénistique achevé de l'époque d'Épaminondas. Comme à Messène, quoique à une échelle moindre, deux montagnes ont été réunies par un mur de défense. Ce mur s'étend sur presque un demi-mile vers Kritsa. La porte, quoique presque enterrée par des glissements de terrain, se distingue très bien. En dessous de ce Palaiokastro se trouve une assez jolie plaine, mais basse et inondée l'hiver ; on l'appelle La Congnia. Depuis Kritsa on peut, en la traversant, rejoindre le Mirambello sans passer par Spinalonga ${ }^{3}$.

3. Bulletin de la Société de Géographie 2e série, III (1935), p. 108-127. I. M. KARAVALAKI, a publié une traduc-

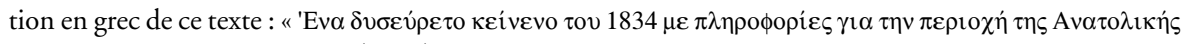

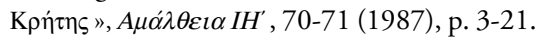


Plusieurs éléments du texte permettent d'identifier avec certitude le site actuel de Latô. Le consul nomme l'endroit Palaiokastro, comme probablement par convention et en l'absence d'éléments plus déterminants, l'ensemble des ruines qu'il a visitées. Du point de vue de la situation générale, la distance d'un mile depuis le village de Kritsa et l'évocation de la plaine de Lakonia nous informent sur l'implantation de la ville antique. La description rapide des ruines nous permet de reconnaitre la porte Ouest, partiellement enfouie, et le mur fortifié qui longe la rue principale menant à l'agora. La chapelle d'Aghios Antonios qui donne à l'époque son nom au lieu, est aujourd'hui encore visible sur le flanc Nord-Ouest de l'acropole Nord. De cette première découverte du site ressortent deux caractéristiques : la première met en lumière l'aspect fortifié et la fonction probablement militaire des vestiges identifiés ; la seconde suggère une continuité d'occupation, depuis la plus haute époque, induite par l'emploi de l'adjectif «cyclopéen ", jusqu’à la période hellénistique. L'évaluation chronologique du consul français montre, de ce point de vue, une connaissance relativement approfondie de l'architecture grecque. L'existence sur le site de phases de construction et d'occupation hellénistiques ne sera pourtant plus affirmée avec autant d'assurance avant les fouilles et les travaux de J. Demargne.

Une vingtaine d'années plus tard, la cité antique est visitée par l'amiral anglais Thomas Abel Brimage Spratt. Au milieu du XIX siècle, il accomplit un périple en Méditerranée orientale, en tant que capitaine du vaisseau Spitfire et sur ordre de la reine Victoria. L'expédition, militaire et scientifique, a pour but d'explorer et de cartographier la région ainsi que d'élaborer une carte maritime de Crète comprenant un relevé des fonds marins. La mission, commencée en mai 1851, se poursuit en 1852, avant d'être interrompue par la guerre de Crimée (1853-1856). Elle reprend en 1859, permettant à l'officier anglais d'achever son voyage en Crète occidentale, à bord du vaisseau Médina. Ses impressions et notes de voyage ont été publiées en 1865 en un volumineux et très intéressant ouvrage ${ }^{4}$, accompagné de gravures élaborées à partir des croquis de Spratt et exécutées par J. Schranz ${ }^{5}$. Le livre a été présenté à l'Académie royale de géographie à Londres, où il fut reconnu comme " a masterly illustration of the physical geography, geology, archaeology, natural history and scenery of the diversified island of Crete ».

4. T. A. B. SPRATT, Travels and Researches in Crete (1865).

5. J. Schranz (1794-1882) est membre d'une famille d'artistes d'origine allemande et installée à Malte. C'est probablement là que T. Spratt fait sa connaissance, puisque l'île est alors la base principale de la flotte royale britannique où Spratt effectuera la plus grande partie de son service. 
Pour visiter le massif du Mirambello, Spratt jette l'ancre dans la rade d'Aghios Nikolaos avant d'être guidéjusqu'à Kritsa, le bourg principal de la région, en suivant la route qui passe par la petite plaine de Lakonia. Une portion de cette route vers Kritsa et Hiérapétra via Kroustas est encore reconnaissable aujourd' hui dans le tracé du caldérim qui mèneà l'Ouest du massif où se trouve Latô. Dans le bref compte rendu qu' il en fait ${ }^{6}$, Spratt décrit à la fois l'environnement général de la ville antique et ses vestiges. La plaine de Lakonia qu'il traverse en venant d'Aghios Nikolaos est caractérisée par une humidité presque permanente qui la rend insalubre et peu propice aux cultures. Son analyse rejoint ici l'observation faite par le consul Fabreguettes une vingtaine d'années plus tôt. Le site du lieu-dit Goulas, dont les ruines sont " in a remarkably good state of preservation ", est implanté sur deux sommets séparés par un ensellement et une profonde dépression qui prend l'apparence d'un cratère : " the site is very singular and interesting from its craterlike form ».

Spratt énumère ensuite les principales caractéristiques de la ville antique, tant du point de vue des techniques constructives que des types d'édifices observables. Le site s'organise sur un réseau de terrasses naturelles ou construites qui permettent l'édification de maisons dont il estime le nombre, important pour une ville antique, à environ deux cents. Les habitations conservent parfois en place les piédroits des portes d'entrée et sont généralement constituées de une à trois pièces. Réparties en plusieurs endroits du site, des citernes alimentent la ville en eau et sont encore recouvertes d'un solide enduit hydraulique. Les murs de la ville et des édifices sont construits en appareil polygonal « cyclopéen » dont le caractère massif s'explique par la qualité du calcaire trouvé sur place. Spratt note que seuls deux édifices témoignent del'emploi d'un appareil rectangulaire à joints vifs. Il insiste à plusieurs reprises sur la qualité de préservation des vestiges et particulièrement de certains murs encore conservés jusqu'à 6 ou 10 pieds de hauteur (environ 1,5 à 3 m). L'uniformité des techniques et du matériau conduit l'auteur à émettre quelques hypothèses de datation : si l'adjectif "cyclopéen ", appliqué généralement aux murs, renvoie aux périodes les plus anciennes des " temps hérö̈ques ", le recours à des blocs plus réguliers semble attester une construction " hellénique " plus récente. Spratt remarque cependant qu'aucun vestige romain ou médiéval ne permet d'identifier une réoccupation tardive du site. Par ses sonorités proches, le toponyme contemporain de Goulas le conduit à proposer l'identification erronée du site avec l'ancienne Olonte, confondue d'ailleurs avec Oléros, et à identifier le sanctuaire de la terrasse avec celui d'Athéna olérienne, tandis qu'il place avec raison la cité

6. T. A. B. SPRATT (supra, n. 4), p. 130-141.

BCH 131 (2007) 
de Kamara à Aghios Nikolaos ${ }^{7}$. Il rejette ainsi l'opinion de R. Pashley ${ }^{8}$ qui voyait en Goulas l'emplacement de la ville d'Arkadia. L'identification lui semble d'autant moins possible qu'Arkadia était réputée pour l'abondance de ses sources d'eau vive dont manque justement le site de Goulas.

Contrairement au compte rendu rapide du consul Fabreguettes, la description de Spratt est illustrée par des croquis et un plan général intitulé « sketch of Goolas9 " (fig. 1). Le relevé est succinct mais permet de situer la voie d'accès à la ville depuis Kritsa, l'emplacement de la gorge qui court au pied Ouest de l'acropole Nord, les caractéristiques topographiques du site et la répartition des vestiges autour de la doline (hollow) ${ }^{10}$. Le texte de Spratt ainsi que son plan d'ensemble marquent le début de l'intérêt pour l'exploration de la cité antique. Beaucoup d'éléments de sa description et de son relevé conditionneront les analyses et documents postérieurs produits par des archéologues, tels quel'emploi de l'adjectif « cyclopéen » et l'identification de Latô à une ville des temps héroïques.

Signalons également que le philologue et archéologue Conrad Bursian mentionne l'existence de ruines au lieu-dit Goulas, ainsi que l'exploration menée par Spratt ${ }^{11}$. Aucune illustration n'accompagne cependant le commentaire général de l'auteur sur cette région de Crète.

7. Kamara est mentionné par l'astronome et géographe Ptolémée ( $\mathrm{I}^{\mathrm{e}} \mathrm{s}$. apr. J.-C.) dans son ouvrage $\Gamma \varepsilon \omega \gamma \rho \alpha-$

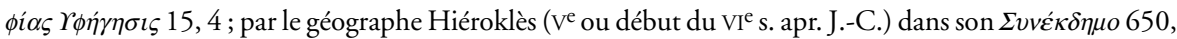

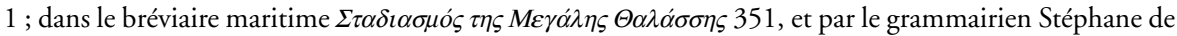
Byzance (VI ${ }^{\mathrm{e}}$ s. apr. J.-C.) dans les $E \theta v \imath \kappa \alpha ́$. Le nom est attesté aujourd'hui dans trois inscriptions : IC I.xvI (Latô), 15 ; IC I.xvI (Latô), 19 ; on ajoutera à ces deux inscriptions une épitaphe trouvée récemment à

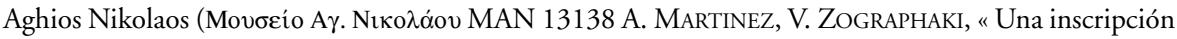
funeraria de Camara (Creta) », ZPE 139 (2002), p. 117-118.

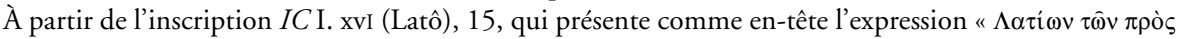

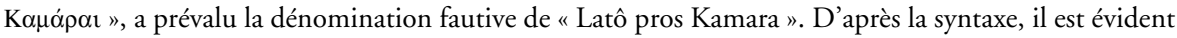
que le texte ne renvoie pas à quelque cité appelée Latô pros Kamara, mais à des Latiens qui habitent à Kamara.

8. R. PASHLey, Travels in Crete, vol. I (1837), p. 231. L'auteur de l'ouvrage n'a cependant pas vu les ruines du lieu-dit Goulas mais semble en avoir entendu parler.

9. T. A. B. SPratT (supra, n. 4), p. 129.

10. La grande dépression (doline) autour de laquelle s'organise le site de Latô sera longtemps appelée " cratère " ou «trou " par les Anglais et les Français. Lidentification des phénomènes karstiques et la nomenclature utilisée pour leur description s'élaborent en effet dans la première moitié du XXe siècle, mais l'utilisation d'un vocabulaire géographique spécifique dans les études archéologiques est un phénomène plus récent. 11. C. Bursian, Geographie von Griechenland, vol. 2 (1868), p. 571-574. 


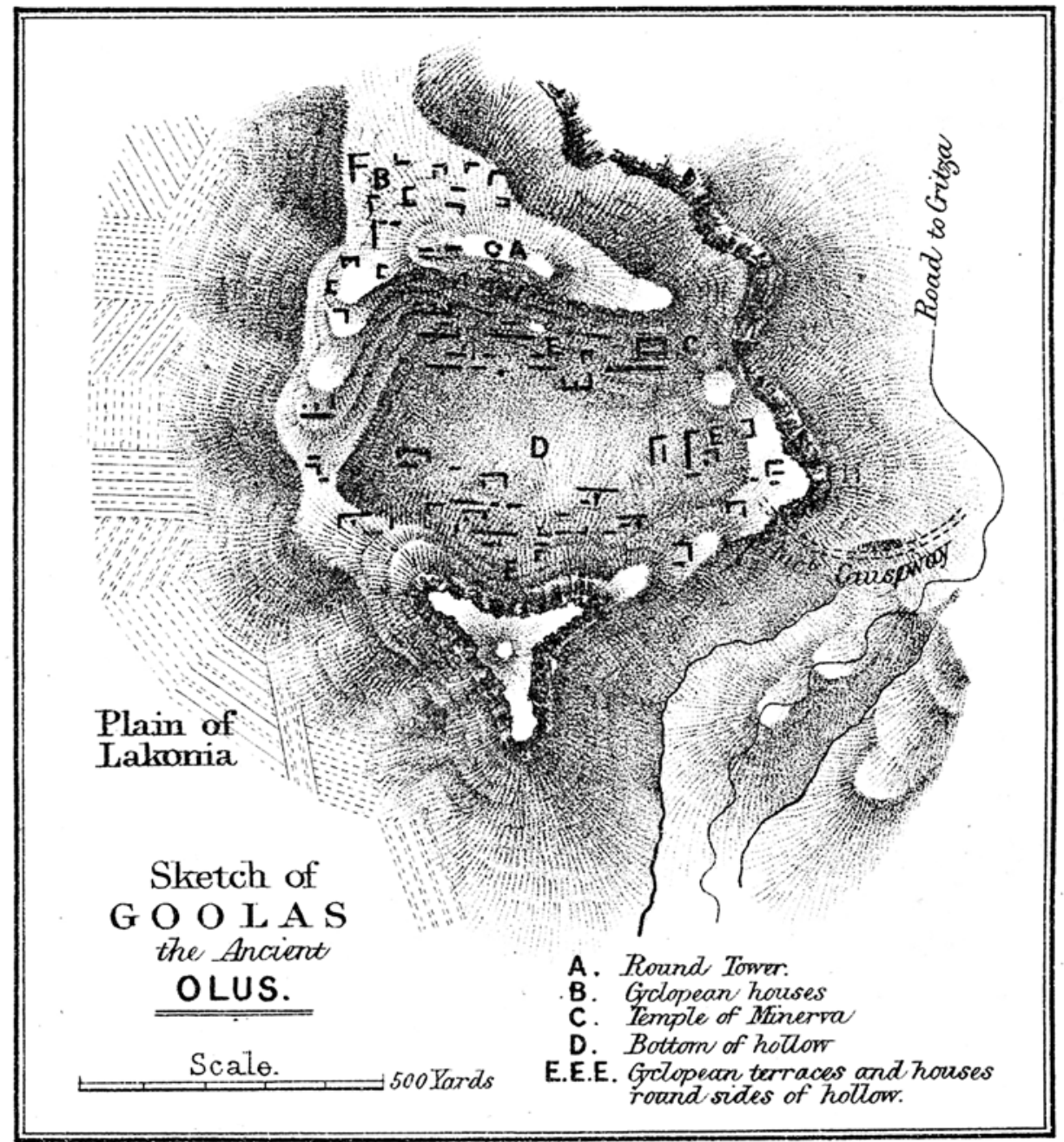

Fig. 1. - Sketch of Goolas. The Ancient Olus, d'après T.A.B. SPRATT, Travels and Researches in Crete (1865), p. 129. 


\section{B) LES ARCHÉOLOGUES ITALIENS À GOULAS/LATÔ}

À la fin du XIXe siècle, plusieurs archéologues italiens prennent le relais des voyageurs en menant les premières études de terrain sur les sites antiques de Crète. L'enthousiasme manifesté dans la description de Spratt attire en effet l'attention des spécialistes sur le massif du Mirambello. Dans les Monumenti antichi dei Lincei, L. Mariani réserve un chapitre conséquent à la description du lieu-dit Goulas ${ }^{12}$. Son exposé complète et enrichit le compte rendu de Spratt en s'appuyant sur des observations personnelles et des remarques rassemblées lors des voyages d'A. Taramelli et F. Halbherr en 1893 et 1894. Insistant sur l'état de conservation des vestiges, leur ampleur et leur qualité, L. Mariani les considère comme les ruines les plus significatives du monde antique : il est le premier à proposer l'identification de Goulas avec la cité de Latô ${ }^{13}$. La spécialisation archéologique du discours se manifeste par la prise de mesures, l'étude détaillée de certains édifices visibles et l'élaboration d'une documentation iconographique variée, rassemblant des croquis, des relevés, des photographies et des copies d'inscriptions. De ce point de vue, elle se démarque nettement de la démarche du voyageur classique : la volonté de rassembler l'ensemble des sources, de mettre à profit des techniques nouvelles et de fournir des explications géologiques, topographiques et historiques à l'implantation du site, est ainsi novatrice. Les informations fournies par Spratt servent pourtant de point de départ et le plan général dessiné par A. Taramelli montre l'évolution d'un document sur lequel s'appuieront J. Demargne et V. Seyk (fig. 2). Le plan est un croquis de petites dimensions, effectué principalement à main levée et orienté au Nord. La route menant à Kritsa et la gorge ( $\phi \alpha \rho \alpha ́ \gamma \gamma \mathrm{l})$ qui court au pied Ouest du site y figurent, ainsi que le tracé approximatif de quelques murs antiques. Les deux acropoles et les terrasses étagées sont rendues par des courbes concentriques qui suggèrent plus nettement les différences de niveau. La situation générale de la ville est exceptionnelle, dans un environnement de moyenne montagne caractérisé par de fortes pentes et par la présence d'une grande dépression autour de laquelle s'organisent les quartiers. L'auteur fournit sur ce point la première explication géologique du phénomène, imputable à une forte érosion et à un écoulement d'eau plus ou moins permanent au fond du "cratère ». La présence de l'eau est figurée par un réseau de petits ruisseaux qui se rejoignent pour se jeter hors de la dépression. Le croquis suggère d'ailleurs que le site est ouvert à l'Est vers Lakonia, alors qu'il est en réalité profondément encaissé de toutes parts et séparé de la plaine par une importante rupture de pente. L. Mariani remarque d'ailleurs que la ville ne possède pas de mur d'enceinte à proprement parler, mais que son système de défense est en grande partie naturel.

12. L. MARIAnI, Monumenti antichi dei Lincei VI (1895), p. 251-282.

13. Ibid., col. 274. Le nom de la cité de Latô était connu des spécialistes dès 1879, voir Th. HomolLE, «Une convention entre trois villes crétoises ", $B C H 3$ (1879), p. 130.

BCH 131 (2007) 


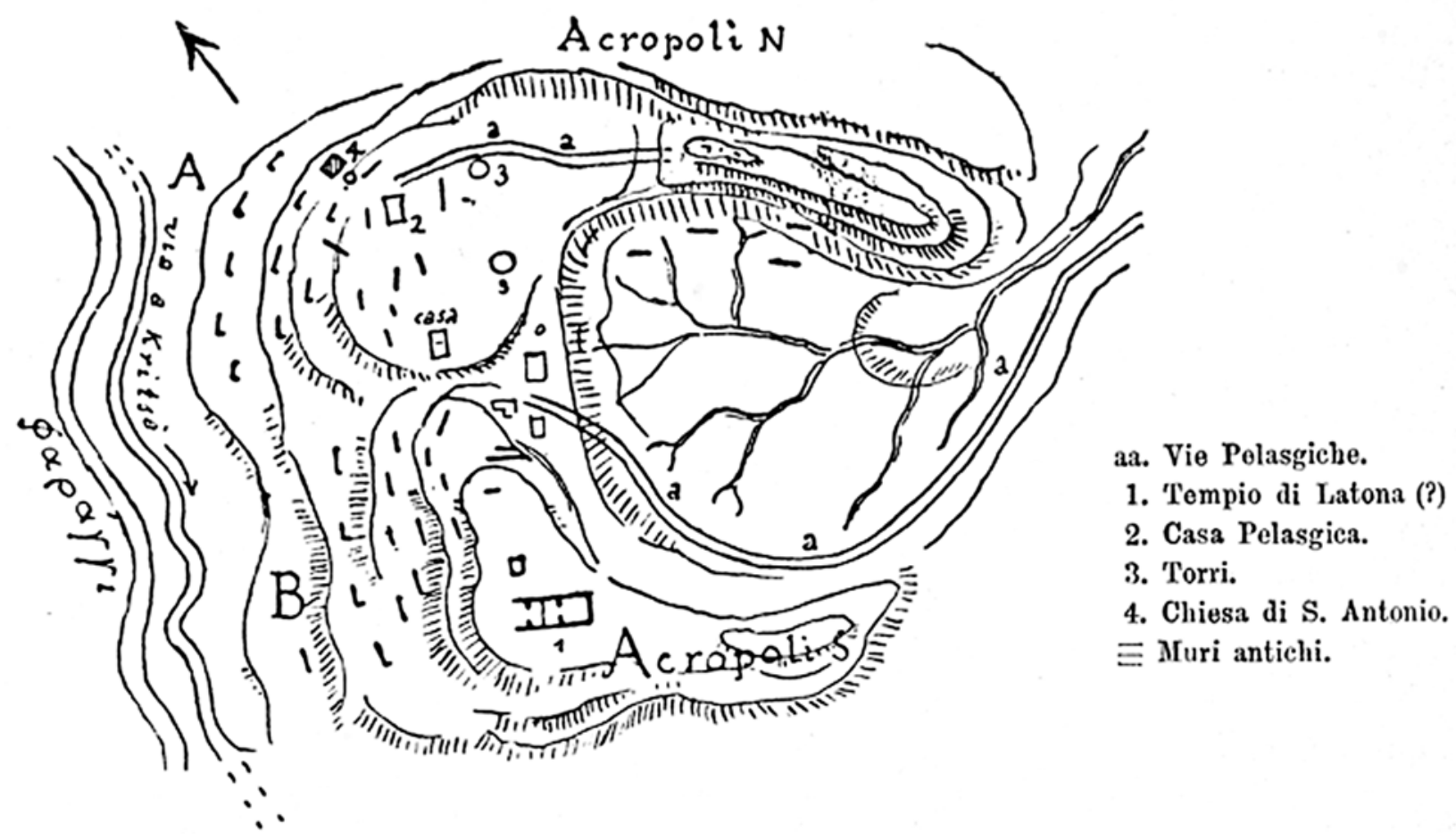

Fig. 2. - Croquis du site de Latô, d'après L. MARIANI, Monumenti antichi dei Lincei VI (1895), p. 251252.

L'étude des savants italiens s'est ensuite particulièrement portée sur les modes de construction et la typologie des édifices observables sur le site. Qualifié à nouveau de « cyclopéen », l'appareil des murs est illustré par des croquis et des relevés. L. Mariani souligne la prédominance de l'appareil polygonal et de blocs de grand module taillés dans le calcaire local. Il évoque également la présence de blocs réguliers, voire rectangulaires, de dimensions plus réduites, qui suggèrent différentes phases de construction, dont les plus récentes pourraient dater de l'époque historique. Le site de Goulas serait ainsi celui d'une ville préhellénique, abandonnée aux temps historiques. Les techniques sont rapprochées à la fois de celles que l'on observe à Tirynthe en Argolide ou à Iouktas en Crète même, et des sites de Kani Kastelli 
et de l'Anavlochos, d'occupation plus récente. L'auteur émet à ce sujet l'hypothèse, largement vérifiée depuis, du maintien d'une tradition constructive forte dans cette région de Crète où la topographie particulière des sites conditionne l'emploi des techniques et des matériaux. L'architecture des maisons reflète la simplicité et la solidité du mode de construction déjà repéré par Spratt. La description de L. Mariani complète les informations du voyageur en y ajoutant un certain nombre de mesures précises concernant la taille des blocs employés ou la dimension des pièces. L'ensemble est illustré par des photographies et des relevés de plans ou de murs en élévation.

L'originalité du texte de L. Mariani se manifeste dans la diversité des documents utilisés pour la description et l'étude du site. Si la datation de la ville relève largement de l'hypothèse protohistorique, les savants italiens semblent déjà soupçonner l'existence d'une chronologie plus récente, suggérée notamment par les modes de construction.

\section{C) A. EVANS ET L'ACROPOLE MYCÉNIENNE DE GOULAS}

La publication récente des archives des voyages d'A. Evans en Crète par A. Brown ${ }^{14}$ éclaire d'un jour neuf le travail accompli par l'archéologue anglais dans la région. Il a visité Latô à plusieurs reprises, en 1894, 1895 et $1899^{15}$. Dès sa première visite en avril 1894, A. Evans écrit : "It is approached from the South by the remains of an ancient road with huge Cyclopean supporting walls». Il fait part de son saisissement à la vue des ruines de Goulas et le terme « cyclopéen » revient fréquemment dans ses descriptions. Les comparaisons qu'il développe vont peser pendant longtemps sur l'identification du site comme un site mycénien : la tour de l'acropole Nord est comparée au cercle des tombes de Mycènes, bien qu' elle ne soit pas ici d'utilisation funéraire ; les édifices du pied de l'acropole sont comparés aux casemates de Tirynthe ; dans l'édifice situé au sommet de l'acropole Sud, Evans reconnaît l'emplacement du palais qu'il rapproche de celui de Troie ; il collecte et achète à Kritsa et dans le Mirambello un grand nombre d'objets, notamment des gemmes gravées, censés provenir de Goulas ou de ses environs immédiats ${ }^{16}$. Il met en avant leur ressemblance avec certaines trouvailles de Cnossos et Mycènes. Enfin, deux phrases résonnent comme une conclusion définitive : "there seems to be no trace of anything Hellenic here (...). What one feels is that here perhaps was really the great original focus of Mykenaean culture ».

14. A. Brown, Arthur Evans's Travels in Crete 1894-1899, British Archaeological Reports. International Series 1000 (2001).

15. Ibid., p. 343 .

16. Pour les objets collectés, cf. J. BoARdman, The Cretan Collection in Oxford, The Dictean Cave and Iron Age Crete (1961) (8 numéros dont 3 de provenance latienne non assurée).

BCH 131 (2007) 
Malgré les fouilles entreprises par J. Demargne à partir de 1899, Evans est resté persuadé de la datation mycénienne de la plupart des vestiges de la ville ${ }^{17}$. S. Reinach s'était fait l'écho de cette conviction en France, dans son article sur la Crète mycénienne paru en 1894 dans la revue L'Anthropologie: Goulas est pour lui aussi « la plus grande des cités mycéniennes que l'on connaisse ${ }^{18}$ ». Cette opinion paraît au demeurant avoir pesé lors des négociations franco-anglaises autour des droits de fouilles à Cnossos et Latô ${ }^{19}$. Lors du voyage d'Evans en Crète en 1899, les relations entre les autorités anglaises et françaises sont tendues sur cette question et l'archéologue semble vouloir user de tous les moyens pour garder la haute main sur un certain nombre de sites, dont Goulas et Itanos. Il écrit à C. D. E. Fortnum le 4 février 1899: «Hope to get away at the end of the month to Crete-where the French are trying to push out English archaeological exploration! They have seized the two places I was trying to secure for the British School at Athens. » Il ajoute dans son journal du 23 mars qu'il compte sur le soutien du Prince George : " Prince George himself very favourable. Promised all we want and even said we might have Goulas, as he has not answered Homolle's request, and would count our claim from date of letter sent on by Duke of York. It was arranged that as the French had asked for Goulas and Itanos, we should have Praesos as well as the other sites reserved to us. " L'affaire se double d'un malentendu concernant les droits moraux d'exploration du site de Cnossos : l'École française d'Athènes manifeste l'envie de réactiver ce droit établi depuis 1891 par A. Joubin ${ }^{20}$, mais arrivé à échéance en 1894 ; A. Evans, avec le soutien financier du Cretan Exploration Fund, compte de son côté effectuer des fouilles à Cnossos dont il a personnellement racheté une partie des terrains. Pour résoudre la question, A. Evans et D. Hogarth cèdent leurs droits sur Goulas. Dans une lettre du 12 avril 1899 envoyée de Delphes et conservée à l'Ashmolean Museum²1, Th. Homolle, alors directeur de l'École française d'Athènes, accepte de céder en échange les droits français sur Cnossos.

17. Même en 1901, Evans n'admet pas la nouvelle datation proposée par J. Demargne, « Mycenian Tree and Pilar Cult and its Mediterranean Relations ", JHS 21 (1901), p. 2 n. 3.

18. S. REINACH, L'Anthropologie (1894), p. 4. A. Evans ouvre également son article paru dans le $A B S A 2$ (1895-1896), p. 169 par cette phrase : «It may safely be said that of no prehistoric city on Hellenic soil are such extensive remains extant above ground as of the Cretan Goulas ».

19. L'ensemble de l'affaire concernant l'échange des droits sur Cnossos et Latô est relaté dans l'ouvrage d'A. Brown (supra, n. 14), p. 270-271 et 296.

20. Th. Homolle, "Nouvelles et correspondances", $B C H 15$ (1891), p. 452, n. 3 ; voir aussi K. CHRISTOFI, "Les Français en Crète. De la huitième question de l'Académie à la concession de Zouroképhalo ", BCH 120 (1996), p. 357-371.

21. Dans la nécrologie de J. Demargne qu'il rédige, A. REINACH fait allusion à cette rivalité entre la France et l'Angleterre : «Il [a] dû, comme Joubin, faute d'argent, renoncer à disputer à Evans - ou à partager avec lui-l'honneur des fouilles de Knossos. ", RA 21 (1912), p. 149-150. 


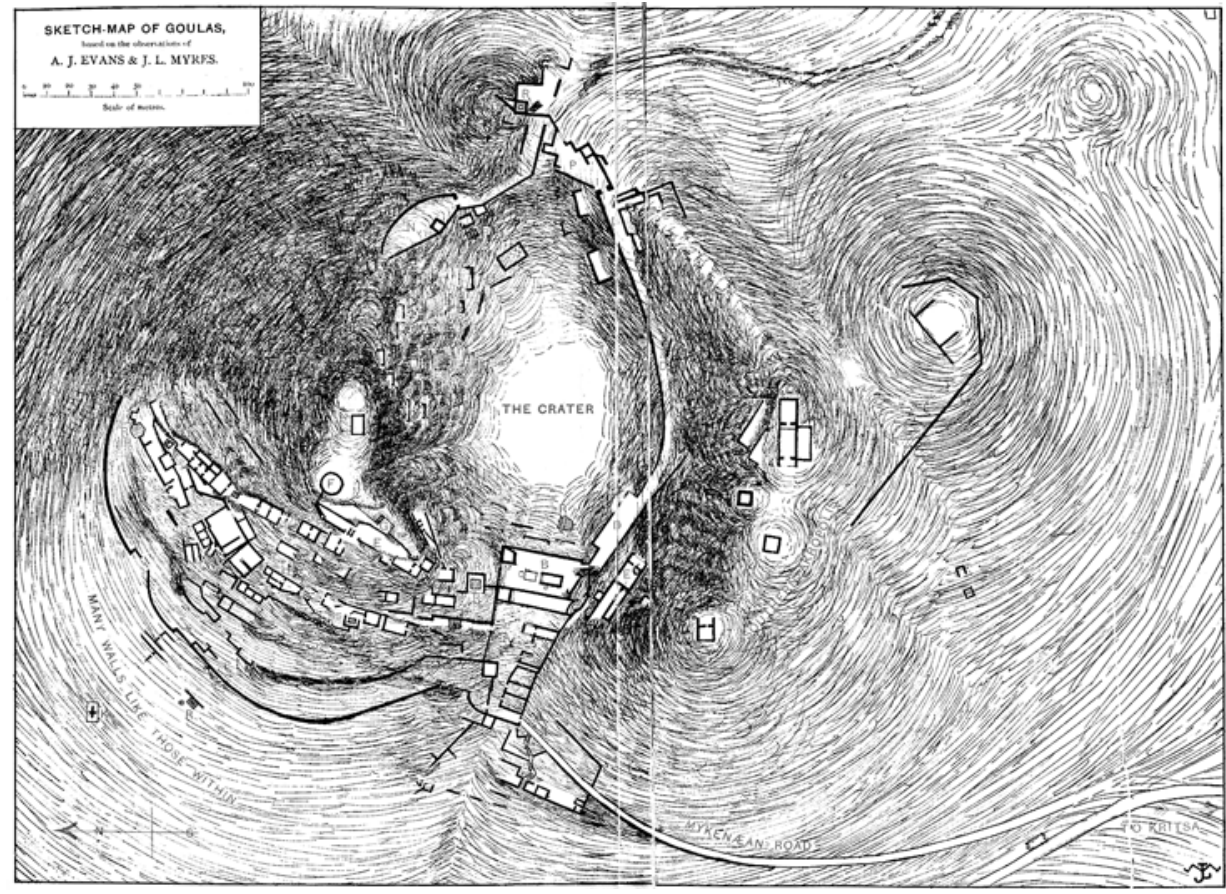

Fig. 3. - Plan général de Latô d’après A. Evans, ABSA 2 (1895-1896), pl. V.

L'étude menée par A. Evans s'accompagne d'un plan général, de photographies et de relevés de certains édifices. Le plan est orienté à l'Est et réalisé par l'architecte et archéologue J. L. Myres (fig. 3). Le relief est rendu à la plume, tandis que l'implantation des ruines est dessinée au trait. Les édifices sont identifiés par des lettres qui renvoient à la désignation de la tour de l'acropole Nord, de maisons, du sanctuaire hypèthre de l'agora ou des portes de la ville. Le plan s'inscrit dans la lignée du croquis établi par Spratt, tout en corrigeant ses erreurs d'orientation et d'identification. A. Evans souligne à ce sujet la difficulté du travail de relevé dans un site aussi accidenté : «It must here be said that, from the vast masses of limestone blocks which encumber the ground, the tangled growth of brush-wood in their 
crevices, and the steepness of part of the site, the planning and measurement of the remains is a work of extraordinary difficulty, nor can anything more than approximate accuracy be claimed for the general result ${ }^{22}$ ». Les descriptions détaillées des principaux monuments directement observables, à savoir le mur fortifié bordant au Nord la rue principale, la porte Ouest, le portique, les bastions du prytanée, l'exèdre Ouest et le temple (identifié comme la maison d'un personnage important), sont illustrées par des croquis en plan, en coupe ou en vue cavalière qui permettent à la fois de situer les vestiges et de caractériser l'appareil des murs. Reconnaissant l'emplacement de l'agora, située à la croisée de plusieurs rues et dans l'ensellement entre les deux acropoles, A. Evans est le premier à proposer un plan général de la rue principale menant de la porte Ouest au point central de la ville.

\section{J. DEMARGNE ET LE PLAN DE L'ARCHITECTE SEYK (A. F., H. W.)}

\section{A) LES CAMPAGNES DE TERRAIN ET L'ÉLABORATION DU PLAN}

À partir de 1899, l'École française d'Athènes s'engage officiellement dans l'exploration du site. La chronologie proposée pour Latô change alors de manière radicale : J. Demargne, chargé de la fouille, affirme ainsi que « tout appartient au contraire à l'époque grecque archaïque ou à une époque postérieure [et que] s'il a existé ici une ville mycénienne, elle a été effacée par une reconstruction totale23 ». De 1899 à 1912, plusieurs campagnes de fouilles sont menées par l'École française d'Athènes. J. Demargne débute ses travaux dans l'été 1899 , avec une première mission en août et septembre, puis une seconde en août 1900, avec l'aide de vingt-cinq ouvriers en moyenne. Il étudie essentiellement la partie centrale du site, mais mène aussi une exploration plus large : « [les fouilles] ont porté sur plusieurs points, en dedans et en dehors de la ville ${ }^{24}$ ». Les conditions d'exploration ne sont pas faciles, comme en témoigne la réaction de D. Hogarth qui visite le site avec Evans le 24 avril 1899; il se déclare « rather dispappointed - walls, walls, walls ! nothing but rought red ware 25 ».

22. A. Evans, "Goulas : the City of Zeus", ABSA 2 (1895-1896), p. 170.

23. J. DemARGNE, «Les ruines de Goulas ou l'ancienne ville de Latô en Crète », BCH25 (1901), p. 306 et 307.

24. Id., "Fouilles à Latô, en Crète 1899-1900 ", BCH 27 (1903), p. 206.

25. A. Brown (supra, n. 14), p. 300. 
Loin d'envier l'entreprise française, Hogarth souligne que la difficulté d'exploration d'un tel site nécessiterait la mise en place d'un système de rails Decauville pour évacuer les déblais ; le coût, élevé, est estimé à environ mille livres et la durée, à deux ou trois ans de travail. J. Demargne commence officiellement les travaux à Latô à l'été 1899, après la promulgation de la loi sur les antiquités en juillet ${ }^{26}$. Il semble pourtant avoir déjà fait quelques observations sur l'acropole Sud au moment de la dernière visite d'Evans (D. Hogarth précise en effet qu'il a déjà « fait des trous (grubbed) »). Pour des raisons de santé, J. Demargne ne peut reprendre les travaux à Latô au-delà de 1900, et meurt de tuberculose à 42 ans, en 1912. A. Reinach lui succède : "C'est à moi, qui ai repris ses fouilles en 1910-11, qu'il incombera de mettre en valeur l'importance des résultats acquis ${ }^{27}$ ». A. Reinach n'a finalement mené qu'une fouille sur le mont Thylakas, à l'Est, où il a découvert un petit sanctuaire 28 et a pratiqué quelques sondages, malheureusement non répertoriés, sur le site de Latô ${ }^{29}$.

\section{B) L'ARCHITECTE V. SEYK}

L'exploration de terrain et l'étude réalisées par J. Demargne s'accompagnent du relevé systématique des ruines visibles sur le site (fig. 4). Si certains vestiges ont fait l'objet de fouilles ou de dégagements, la plupart d'entre eux ont donc été dessinés sans doute après un simple nettoyage. L'entreprise se démarque sur ce point d'autres études contemporaines, ou postérieures, menées à l'échelle de villes entières, à Olynthe et à Théra ${ }^{30}$, où le relevé ne concerne que les vestiges fouillés. Le relevé exhaustif de la ville est mené en collaboration avec l'architecte V. Seyk, dont J. Demargne fait un éloge sans réserve : « La carte et le plan qui sont reproduits ici (planche XX-XXI) sont l'œuvre d'un architecte, M. Seyk ;

26. J. Demargne, BCH 27 (1903), p. 206 : «Le gouvernement crétois, après avoir promulgué sa loi des antiquités dans les premiers jours de juillet 1899 , m'autorisa à faire des fouilles au nom de l'École française à Goulas et à Itanos. Les fouilles commencèrent à Goulas la même année et eurent lieu pendant les mois d'août et de septembre. Elles furent reprise en 1900 et poursuivies pendant le mois d'août. »

27. RA 21 (1912), p. 150.

28. A. ReINACH, «L'autel rustique du Mont Phylakas ", RA 22 (1913), p. 278-300. On trouve aussi dans cet article quelques remarques sur le massif montagneux dans son ensemble, avec une mention du hameau de Melissakia.

29. P. DEMARGNE signale que certaines figurines publiées dans "Terres-cuites archaïques de Latô (Crète) ", BCH 53 (1929), p. 382, proviennent « des sondages complémentaires exécutées en 1910 par Adolphe Reinach".

30. D. M. Robinson, J. W. Graham, The Hellenistic House. Excavations at Olynthus VIII (1938) et D. M. RobInson Domestic and Public Architecture. Excavations at Olynthus XII (1946) ; W. DÖRPFELD, Thera I (1899) et F. HILlER VON GAERTRINGEN, Thera III (1904). 
ils ont été dressés pendant l'été 1900. Malgré certaines imperfections, le plan est le meilleur qui existe de Goulas, il annule les précédents; il donne une idée exacte de l'étendue des ruines et bien que M. Evans ait parlé de leur « masse presque inépuisable », nous pouvons assurer qu'il n'y a aucune partie de la ville ancienne qui ait échappé aux investigations de son auteur ${ }^{31}$ ». Malgré l'important travail fourni à Latô et l'hommage rendu par J. Demargne, le nom de V. Seyk n'apparaît pas sur le plan publié que l'on désigne plus souvent comme le "plan Demargne » que comme «plan Seyk ». L'architecte Seyk n'est guère connu aujourd'hui que des spécialistes d'archéologie homérique. Il est l'auteur de l'ouvrage Das wahre und richtige Troja-Ilion paru à Prague en 1926 et dans lequel sont reproduits trois plans. V. Seyk, pourtant collaborateur de W. Dörpfeld, y réfute l'identification de la Troie homérique avec Hissarlik. L'archéologue Ch. Vellay a popularisé cette hypothèse en France en publiant un plan d'Hissarlik réalisé par Seyk ${ }^{32}$. Avant cette période, son nom est attaché au site de Palaikastro dont il dessine un plan accompagnant le rapport de fouilles : il s'agit en réalité d'un relevé « redrawn from the Dawkins' plan by Herr Seyk, draughtsman to the German Archaeological Institute at Athens "33. V. Seyk est donc un dessinateur lié à l'Institut allemand d'Athènes. Sa présence aux côtés de J. Demargne lors des missions latiennes s'inscrit dans l'histoire des collaborations techniques fréquemment pratiquées à l'époque par les équipes étrangères qui travaillent en Grèce. Cette pratique régulière a été inaugurée à l'École française d'Athènes par Th. Homolle, directeur de 1890 à 1903, et mise en œuvre à Délos et à Delphes notamment ${ }^{34}$. En revanche, on ne trouve aucune mention de Seyk dans les fouilles de Troie, menées sous la direction de W. Dörpfeld en 1893-189435, ou dans celles de Tirynthe. Architecte et dessinateur, Seyk fut donc un collaborateur de l'Institut allemand qui participa occasionnellement à des travaux de relevé ou de mise au net pour d'autres institutions. C'est dans le cadre de ces collaborations ponctuelles qu'il travailla à la réalisation du plan de Latô.

31. J. Demargne, BCH 25 (1901), p. 286.

32. Ch. Vellay, Les nouveaux aspects de la question de Troie (1930), p. 89 ; Ch. VellaY, Controverses autour de Troie (1936), p. 146 et p. 157.

33. R. C. BosanqueT, "Excavations at Palaikastro II ", ABSA 9 (1902-1903), p. 277.

34. M.-Chr. HellmanN, «Les architectes de l'École française d'Athènes ", BCH 120 (1996), p. 191-222.

35. On ne trouve pas le nom de Seyk dans W. DöRpFELD, Troja und Ilion (1902) : le plan est produit par W. Dörpfeld et W. Wilberg. 


\section{C) UNE RÉALISATION D'AMPLEUR POUR L'ÉPOQUE}

La disparition des archives de J. Demargne ne nous permet pas d'évaluer avec précision l'ampleur des travaux menés sur le terrain, de localiser avec certitude les édifices dégagés, voire fouillés, ou de mesurer les difficultés rencontrées dans l'élaboration des relevés. La fréquentation régulière d'un site aussi accidenté et rude que celui de Latô nous fait toutefois apprécier à sa juste valeur la grande qualité du travail réalisé à l'époque. Nous ne connaissons pas non plus les conditions exactes dans lesquelles V. Seyk a élaboré le plan $\mathrm{du}$ site, mais ce document représente une prouesse remarquable à l'aube du XXe siècle. Il s'agit en effet du premier plan complet d'une ville grecque, connue sans fouille d'ampleur en raison du bon état général des ruines signalé par tous les visiteurs, de Spratt à Evans. La tâche fut d'autant plus difficile à mener dans ce lieu escarpé que J. Demargne et V. Seyk n'ont bénéficié d'aucun équipement sophistiqué, contrairement à Olonte où J. Demargne avait fait appel à la marine française ou à Délos où la carte de l'île a été réalisée par le capitaine A. Bellot avec du matériel militaire ${ }^{36}$. L'armée française, pourtant encore en station dans cette partie de la Crète, n'a manifestement pas été sollicitée dans le cadre de cette mission. Le plan constitue ainsi un document d'une qualité indéniable qui, comme l'a dit J. Demargne " annule les précédents ». A. Evans et D. Hogarth avaient déjà en leur temps souligné la difficulté qu'il y avait à relever des vestiges dans un site aussi accidenté. J. Demargne insiste également sur ce point dans le premier article de synthèse qu'il publie: "Ceux qui connaissent les lieux et qui se sont risqués sur ces pentes glissantes et sur ces entassements de pierres apprécieront la patience et l'endurance qu'il a fallu pour mener à bonne fin ce travail ${ }^{37}$ ".

Le plan de Seyk constitue ainsi le document planimétrique le plus complet réalisé et publié jusqu'alors, par l'ampleur de la zone relevée et le détail des murs, des rues, des citernes et des indications géographiques. Malgré ses qualités, le plan n’a cependant pas été largement diffusé à travers la bibliographie concernant l'urbanisme ou l'architecture des cités antiques : il a certes été repris dans quelques synthèses générales sur l'histoire de la ville ${ }^{38}$, mais il a curieusement été peu utilisé dans les études spécialisées sur la ville

36. A. BeLlot, Carte de l'âle de Délos au 1/10 000 avec un commentaire explicatif, EAD I (1909).

37. J. Demargne (supra, n. 23), p. 286.

38. M. Pallottini, Alle origini della citta europea, Storia dell'urbanistica (1993), p. 148, fig. 83 et p. 149 où Latô est encore donnée comme une ville protohistorique.

BCH 131 (2007) 
grecque. Le plus souvent, le site n'est mentionné que pour son agora ${ }^{39}$ ou son prytanée ${ }^{40}$. Le plan a été toutefois repris dans le Guide des fouilles françaises de Crète et habillé, pour la circonstance, de courbes de niveau ${ }^{41}$. L'explication de cette rareté d'utilisation peut tenir à diverses raisons circonstancielles :

- d'une part, les fouilles de Délos ont fourni presque au même moment l'exemple d'une ville hellénistique bien conservée, relativement complète et fouillée en profondeur. Le plan d'une ville dont seule une très faible partie avait fait l'objet d'une fouille systématique n'a peut-être pas paru assez fiable pour pouvoir être considéré comme un document assuré. On peut également ajouter que les ruines de Latô, si impressionnantes soient-elles, ont sans doute faiblement pesé face à la richesse et à la variété des vestiges de la Délos hellénistique ;

- d'autre part, l'actualité archéologique en Crète est alors essentiellement minoenne et les sites de Palaikastro et Gournia fournissent à la même époque des plans de ville ou de quartiers. De même dans les Cyclades, à Phylakopi de Mélos où la ville est fouillée entre 1896 et 1899 et publiée en détail en $1904^{42}$.

\section{D) LES FOUILLES ET LES RELEVÉS EFFECTUÉS ENTRE 1967 ET 1971}

Après plus d'un demi-siècle d'abandon, le site de Latô est à nouveau l'objet de travaux de recherche et de fouilles menés de 1967 à 1971 par Olivier Picard, Pierre Ducrey et Vanna Hadjimichali. L'équipe, pluridisciplinaire et internationale, est assistée par deux architectes, Odette Farner et Martin Schmid, ainsi que par le topographe D. Petropoulos. La reprise des travaux est soutenue et encouragée par les autorités politiques et archéologiques de la région d'Aghios Nikolaos qui participent désormais activement et financièrement à la mise en valeur du site ${ }^{43}$. Les missions ont pour but essentiel d'éclairer des questions laissées en suspens depuis les campagnes de J. Demargne, notamment celle de l'organisation

39. F. Tritsch, «Die Stadtbildungen des Altertums und die Griechische Polis », Jahres. XXVII (1932), p. 183. L'article comprend un plan de l'agora de Latô et non de la ville entière ; les plans de ville proposés sont ceux de Phylakopi, Gournia ou Palaikastro. R. MARTin, L'urbanisme dans la Grèce antique (1956), p. 81, où Latô et Dréros " peuvent être considérés comme l'expression urbaine de la Crète dorienne "; p. 82 : " ces villes-forteresses reflètent l'état incertain de la Crète (...) "; R. MARTIN, Recherches sur l'agora grecque: études d'histoire et d'architecture urbaines (1951), p. 226, fig. 25.

40. Parmi d'autres exemples, C. WEICKERT, Typen der Archaischen Architektur in Griechenland und Kleinasien (1929), p. 174, n. 1 ; N. MAC Donald, The Political Meeting Places of the Greeks (1943), p. 32-34.

41. Cl. Tiré, H. VAn EfFenterre, Guide des fouilles françaises en Crète, SitMon II (1983), p. 101, fig. 27.

42. Excavations at Phylakopi in Melos, ABSA Suppl. Papers 4 (1904). T. D. Atkinson, architecte, en fait le relevé en 1899.

43. P. Ducrey, O. PiCARD, « Recherches à Latô VII. La rue Ouest. Habitations et défense », BCH 120 (1996), p. 721. 
du centre civique et religieux de Latô. De ce fait, les fouilles et les relevés sont localement limités, à la fois par les objectifs fixés et par les conditions cadastrales particulières du site : Latô est en effet toujours exploitée à l'époque pour la culture des amandiers et les terrains sur lesquels est établie la ville ne relèvent pas tous de la propriété publique.

Les fouilles et les études de bâtiments répondent à plusieurs objectifs. Pour cerner l'organisation du système défensif de la ville et son réseau de circulation, des recherches sont menées dans le secteur de la porte Ouest et de la rue Demargne. Plusieurs maisons fortifiées sont dégagées et alimentent aujourd'hui encore notre connaissance de l'habitat latien. Les caractéristiques de l'habitat sont également mises en valeur par l'analyse de maisons déjà dégagées et par la fouille stratigraphique de nouveaux exemples, à l'instar de la maison du Temple située sur une large terrasse au pied de l'acropole Sud. Dans ce même secteur, l'analyse approfondie des vestiges conduit au dégagement du théâtre et de l'exèdre, enfouis sous un pierrier et sous les anciens déblais des fouilles de J. Demargne. Les recherches menées sur l'emplacement du temple mettent au jour trois fours de potier archaïques dont le matériel documente l'histoire de Latô et de sa production céramique. Enfin, des études sont conduites sur le prytanée, repéré par A. Evans et fouillé par J. Demargne, afin d'en affiner la chronologie.

L'ensemble de ces travaux permet l'établissement d'une documentation nouvelle et fournie qui s'appuie notamment sur les progrès techniques réalisés depuis le début du siècle dans les domaines de la photographie, de la topographie et de l'architecture. Les relevés sont systématiquement cotés, pour pallier l'absence des courbes de niveau dans le plan réalisé par V. Seyk et J. Demargne. La rue principale de la ville est également rendue en coupe, permettant de visualiser à la fois l'appareil du mur fortifié qui la borde au Nord, et la déclivité de la pente sur laquelle elle est aménagée. Un certain nombre de relevés sont effectués en pierre à pierre et non plus simplement au trait, pour apprécier plus finement la construction des murs et les modules des blocs utilisés. Le critère de la dimension des blocs est d'autant plus important qu'il a constitué longtemps le seul élément d'appréciation et de datation de l'appareil «cyclopéen » de Latô. Les documents figurés sont également illustrés par une riche série photographique de qualité, réalisée en noir et blanc et régulièrement référencée par l'emploi d'un jalon. La documentation produite nourrit ainsi de manière précise la connaissance du site et de son implantation, les caractéristiques constructives des édifices, ainsi que l'évolution diachronique de son occupation.

Les résultats des cinq années de campagne réalisées par O. Picard, P. Ducrey et V. Hadjimichali ont fait l'objet de nombreux articles que nous signalons pour mémoire ${ }^{44}$. Les

44. P. DuCReY, O. PICARD, "Recherches à Latô ", BCH 93 (1969), p. 792-822 ; "Recherches à Latô II. Le grand temple ", BCH 94 (1970), p. 567-590 ; "Recherches à Latô IV. Le théâtre », BCH 95 (1971),

BCH 131 (2007) 
recherches menées ont non seulement permis d'éclairer l'architecture de certains édifices comme le temple, le prytanée ou les habitations, mais elles ont aussi traité plus largement de l'histoire de la ville, de son monnayage, de son matériel céramique et de ses programmes édilitaires mis en œuvre à l'époque hellénistique. Du point de vue de la morphologie urbaine, les études ont porté sur l'organisation de la défense qui utilise à la fois les ressources naturelles de l'escarpement du site et la construction du mur fortifié longeant la rue Ouest, dans lequel sont construites des maisons tours qui permettent, le cas échéant, de barrer le passage vers les terrasses menant aux quartiers d'habitation. L'essentiel de l'étude archéologique a ainsi porté sur le centre politique et religieux de la cité et non sur la ville entière. Les différentes missions ont permis d'élaborer une documentation nouvelle concernant le détail de certains édifices, mais en matière de relevé général, le plan de V. Seyk est resté le document de référence.

\section{LE PLAN SEYK ET LA TOPOGRAPHIE DE LATÔ AUJOURD'HUI (A. F., L. F., H. W.)}

La reprise récente des travaux à Latô oblige à se poser la question de l'exactitude des informations contenues dans le plan Seyk. La difficulté est triple: d'une part, nous ignorons dans quel système topographique le relevé des vestiges a été réalisé ; d'autre part, le relief, qui constitue pourtant un des traits originaux du site, est représenté de manière très schématique et très limitée; enfin, l'état du site aujourd'hui n'est plus celui de 1900 : des routes, des constructions, des fouilles en ont modifié l'aspect. Il apparaît donc nécessaire désormais de faire un bilan critique de ce document afin d'en préciser les conditions d'exploitation et de mesurer, par les moyens de la topographie moderne, le degré de fiabilité du plan Seyk.

\section{A) LES APPORTS DU DOCUMENT EXISTANT}

Le plan élaboré par V. Seyk et J. Demargne rassemble d'emblée un certain nombre d'informations précieuses qui font de ce document une base de travail irremplaçable. Il fixe

p. 515-531 ; "Recherches à Latô V. Le prytanée », BCH96 (1972), p. 567-592 ; « Recherches à Latô VI.

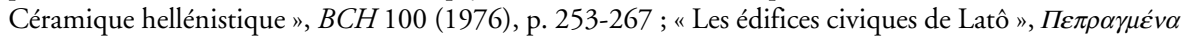

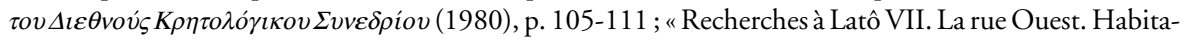
tions et défense ", BCH 120 (1996), p. 721-754 ; V. HADJIMICHALI, " Recherches à Latô III. Maisons ", BCH 95 (1971), p. 167-222. 
notamment la toponymie moderne du site, telle qu'on la connaissait probablement jusqu'au milieu du XXe siècle. Les noms des différents lieux-dits qui jalonnent le site et ne sont pas conservés sur les cartes au 1/15000 ou au 1/5000, nous permettent de différencier aujourd'hui encore les quartiers de Latô (fig. 4).

Si les fouilles et dégagements de J. Demargne n'ont manifestement concerné qu'une petite partie des vestiges enfouis, et plus précisément ceux qui s'étendent depuis la porte Ouest jusqu'à l'agora, le plan, en revanche, a été établi à l'échelle du site entier. La démarche prend d'autant plus de valeur qu'elle correspond à l'intérêt majeur du site de Goulas souligné par tous les visiteurs du XIXe siècle : une ville antique apparemment complète et dont les ruines sont en grande partie observables sans fouilles d'envergure. En l'absence de courbes de niveau, J. Demargne a fait preuve d'une minutie particulière pour signaler les caractéristiques topographiques du site. Le plan contient quelques mentions d'altitude, pour les sommets des deux acropoles et du pyrgos, ainsi que pour le fond de la doline et l'emplacement de l'agora. Les précisions du relief, qu'il s'agisse d'une « pente raide », du « ravin » ou de " rochers à pic " permettent de constater que le site est naturellement protégé sur les côtés Est, Nord et Ouest. On notera cependant une erreur d'appréciation dans la localisation de l'acropole Sud notée à l'emplacement du pyrgos. La répartition des deux acropoles et de ce troisième sommet est pourtant traitée avec justesse dans le texte des deux articles écrits par J. Demargne. Ce dernier a également pris en compte l'implantation plus large de Latô et de son terroir en explorant les environs immédiats de la ville dont il fournit une carte (fig. 5). Il est ainsi le seul à repérer et à localiser les quatre dolines qui se succèdent au SudEst de la ville, sans pour autant identifier le phénomène géomorphologique à l'origine de leur formation. Ses prospections le mènent sur toutes les traces des chemins antiques dont il conserve soigneusement les axes ou les tracés sur le plan ; il repère aussi les ruines de moulins modernes et du fort situé à l'Est que nous avons pu retrouver, ainsi que les vestiges du hameau de Sta Melissakia dont le toponyme n'est plus reporté sur les cartes actuelles. Audelà du plan d'une ville, J. Demargne semble avoir mesuré l'emprise de la cité antique sur son environnement qui, en retour, influe sur son organisation.

Le plan Seyk nous montre également un état de Latô qui n'est, en partie, plus observable aujourd'hui, eu égard aux dégradations naturelles et aux activités humaines qui se sont poursuivies sur le site et à ses abords. Plusieurs facteurs sont à l'origine de la dégradation de certains secteurs ou du caractère illisible de certains vestiges.

- L'ampleur de la végétation sur l'ensemble du site constitue sans doute la cause principale du manque de lisibilité actuelle des ruines. Cette végétation foisonnante provient notamment de l'abandon des terrasses cultivées autrefois par les habitants de Kritsa et plantées d'oliviers, d'amandiers et de pistachiers lentisques dont les rejets sauvages envahissent les pentes et compliquent sérieusement l'observation. Le quartier de Badéna, situé à l'Ouest de la ville et de part et d'autre du chemin moderne qui mène à la chapelle d'Aghios Antonios, est ainsi noyé sous une végétation touffue. Quelques 


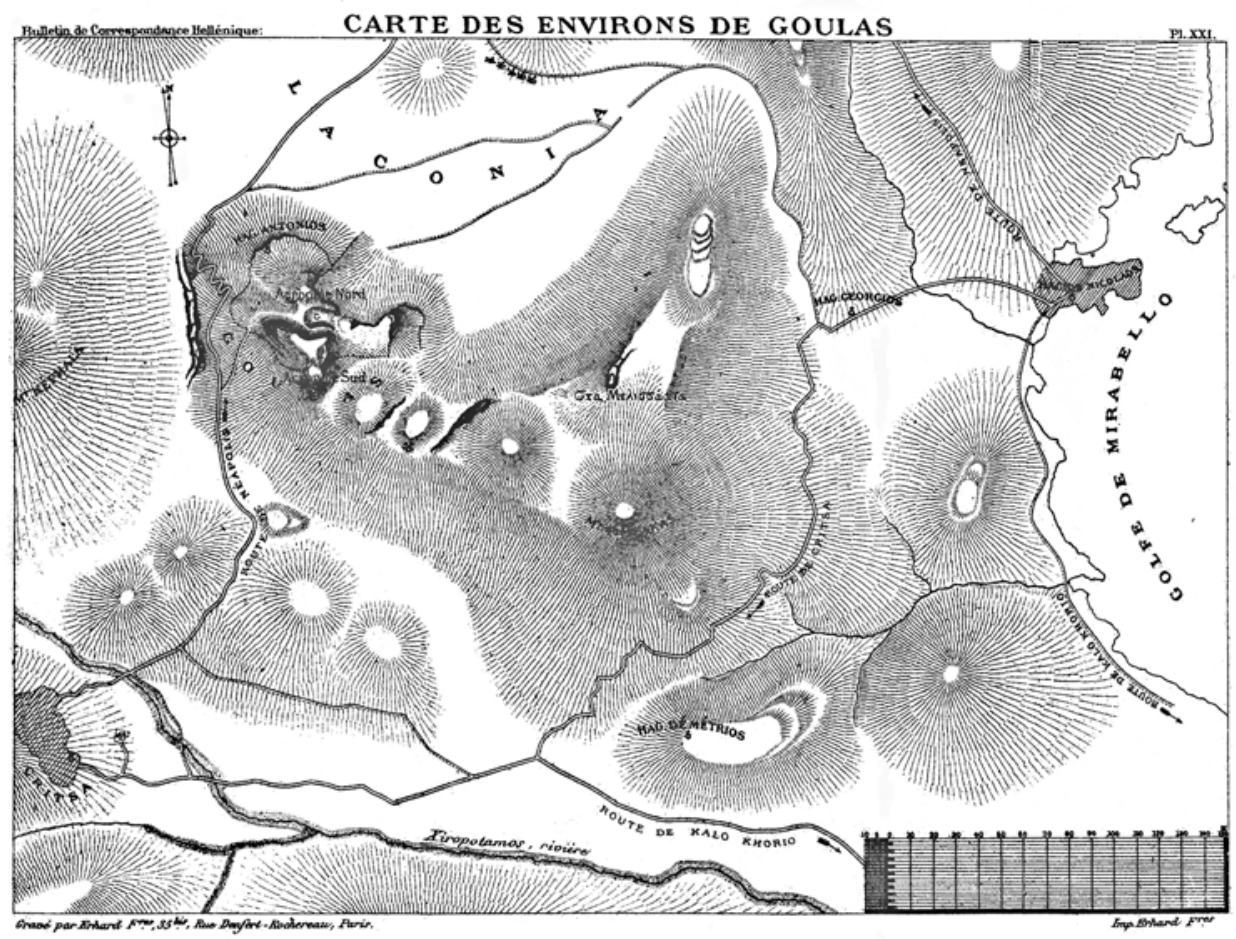

Fig. 5. - Carte des environs de Goulas, d'après J. Demargne, V. SeYK, BCH 25 (1901), pl. XX (EFA).

murs, difficiles d'accès, ainsi que des citernes, ont pu être repérés, mais sont presque entièrement masqués. La même remarque peut être faite à propos des quartiers d'habitation construits sur les parois intérieures de la doline aujourd'hui envahies d'arbres aux proportions parfois impressionnantes (fig. 6).

- Les nombreux éboulements ont également contribué à modifier la lisibilité du site depuis les explorations de J. Demargne. Ils sont majoritairement provoqués par le dénivelé des pentes qui peut atteindre jusqu'à $60 \%$ dans le quartier Nord. Pourtant, c'est essentiellement dans la doline et dans le bas des quartiers Ouest et Nord que le phénomène semble le plus fréquent. La poussée des terres y est en effet plus importante et le substrat plus friable et plus meuble, d'après les observations que nous avons menées sur le terrain. De manière générale, les éboulis se conjuguent avec l'emprise grandissante 


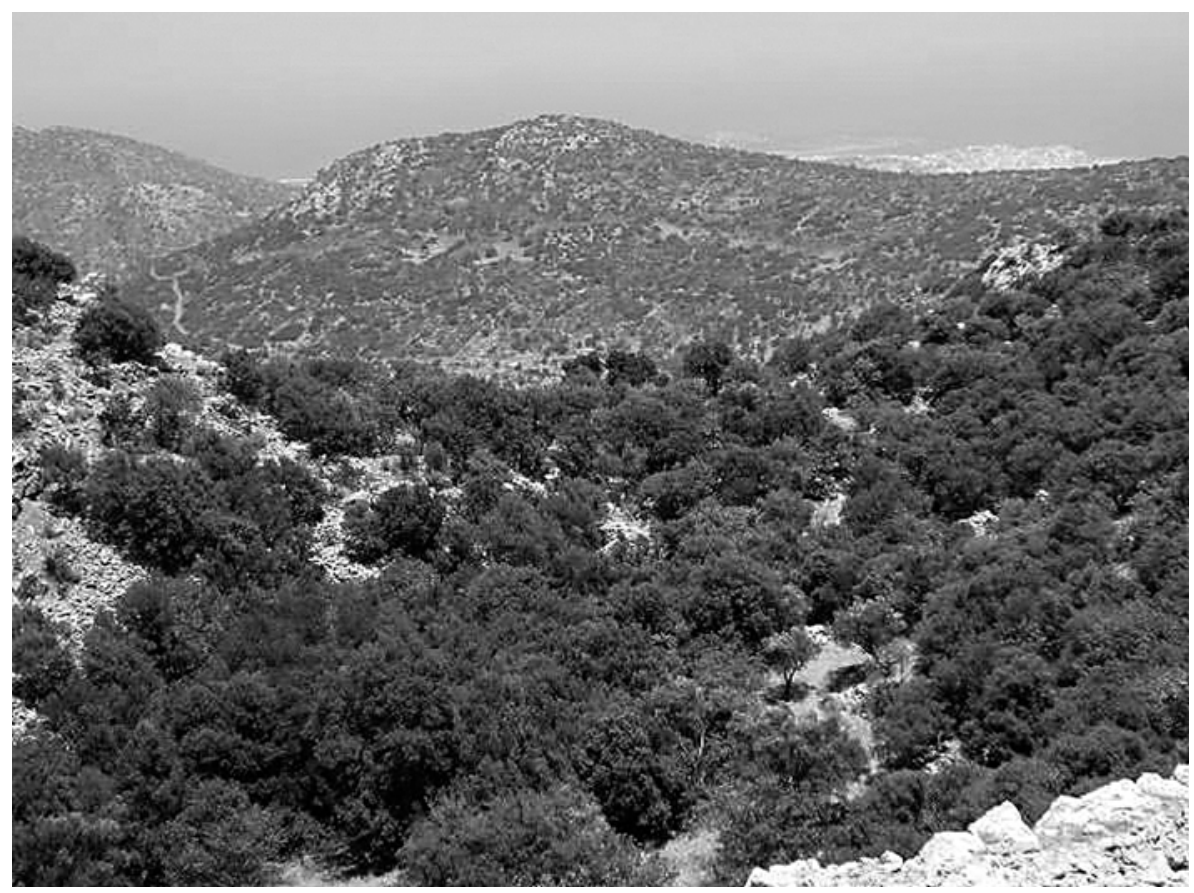

Fig. 6. - Fermeture de la doline à l'Est : des vestiges sous les arbres (cliché H. Wurmser, EFA).

de la végétation pour créer les conditions favorables à la destruction des structures les plus fragiles. La fréquentation du site sur trois ans et à des saisons différentes nous a permis de mesurer la lente mais continue dégradation des ruines dans certains secteurs. La situation du lieu-dit pyrgos semble être plus complexe. Si sa localisation topographique est claire, les vestiges des édifices, déjà largement restitués dans le plan Demargne, n'apparaissent plus qu'en filigrane. Une partie des blocs a pu disparaitre dans la rupture de pente située directement au Sud du bastion, tandis que d'autres blocs ont pu être remployés dans diverses constructions modernes, eu égard à l'accessibilité du secteur et à sa proximité avec le village de Kritsa.

- L'évolution constante de l'état du site est également imputable aux ponctuelles, mais régulières, réoccupations de Latô au cours $\mathrm{du} \mathrm{XX}^{\mathrm{e}}$ siècle. La dernière réoccupation importante du site date de la deuxième guerre mondiale, durant laquelle les habitants de Kritsa se sont réfugiés à Latô en occupant des édifices existants et en construisant une aire à battre dans le portique de l'agora. La construction de cette aire et d'une structure similaire dans le quartier d'Agaratiès à l'Est, documente l'existence de cultures céréalières relativement importantes et exploitées au moins jusqu'à la deuxième moitié du 


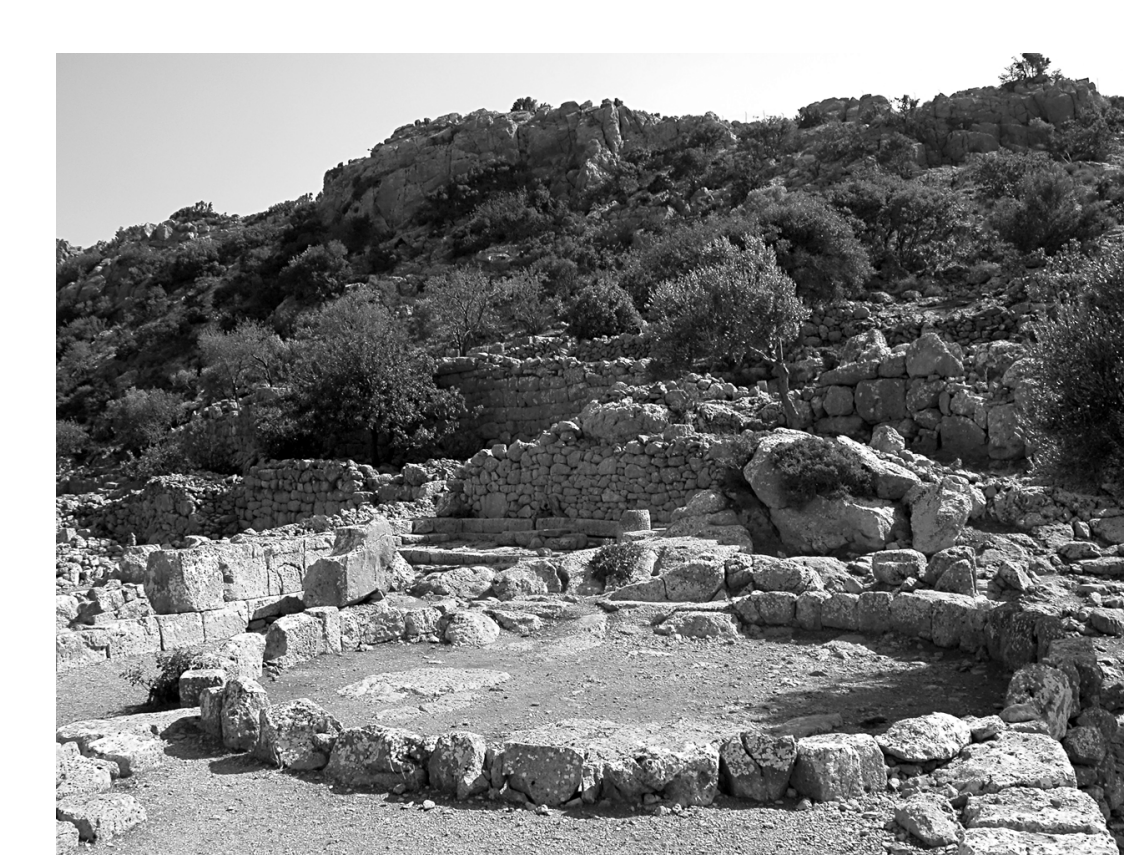

Fig. 7. - Aire à battre modern

xxe siècle à proximité du site (fig. 7$)$. On note également qưune garnison allemand s'était établie sur le mont Thylakas.

Au-delà de cette période de conflit et malgré lexpansion du village de Kritsa, Latô a toujours servi à la culture des amandiers et d autres arbres fruitiers. Les fouilles effecteuespar O. Pcard, P. Ducrey et V. Hadjmichali la la fin des annéess soixante ont dû ain des opérations témoignent d' une activité agricole menée jusqu'aux abords du prytané et sur la terrasse du temple. Des réoccupations legereses ont eu lieu tout au long du siecle. notamment avec la construction de cabanes qui servaient aux besoins agricoles. végetation abondante a aussi fait de Censemble du secteur un lieu de páture privilégié aujourd'hui encore par les bergers de la région : les bêtes viennent pâtre à heure
régulière, malgré la clôture installée par le service archéologique De nombreux enclos quadrillent notamment le secteur Est de la ville et rendent difficile la lecture des vestiges: Iláéccupation du secteur r'Agaratiès par des bergeriesese des enclos a en effet
profondément perturbé les conditions d'observation des ruines. Il est ainsi devenu 


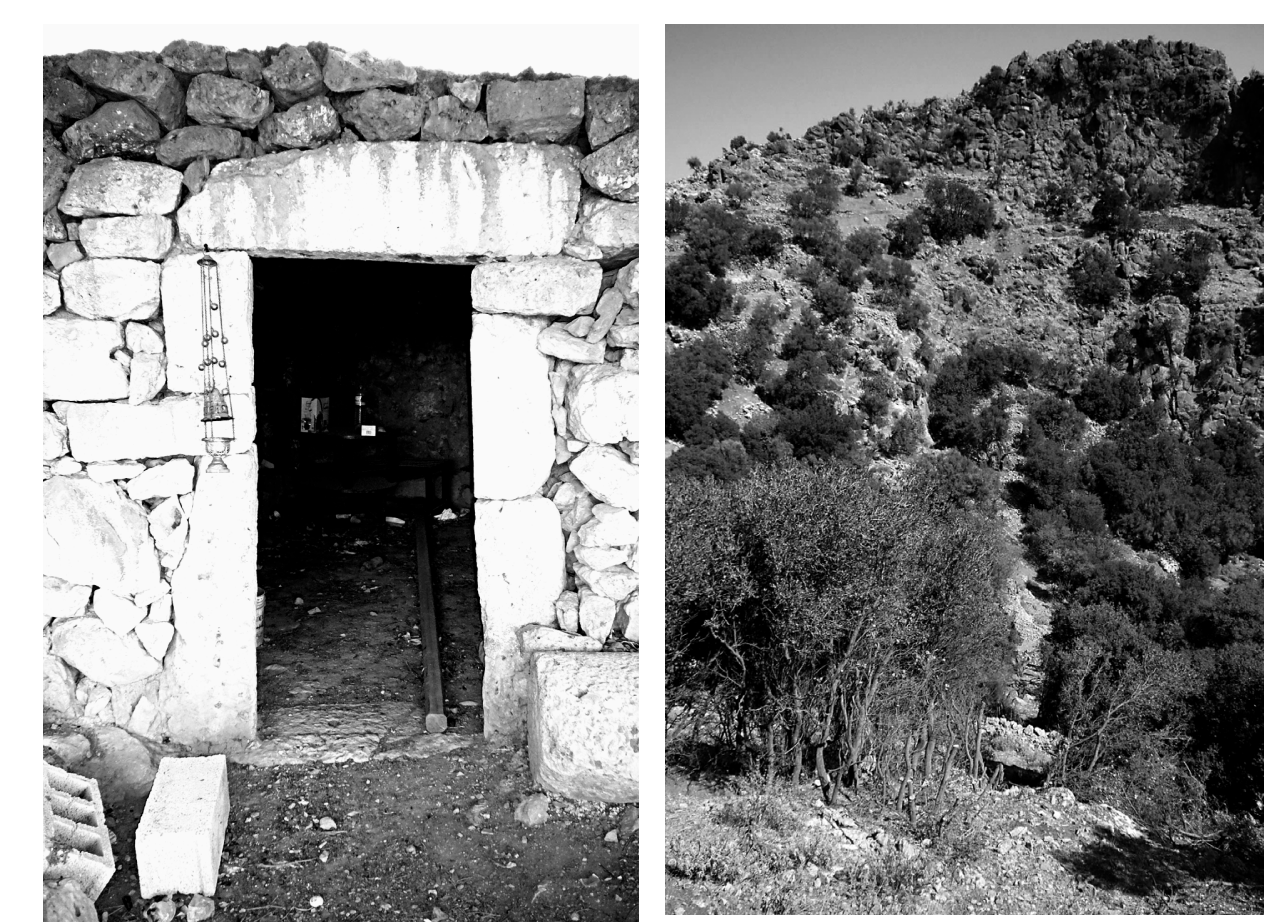

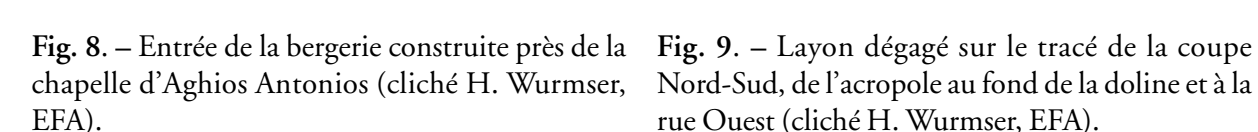

presque impossible de décrypter l'enchevêtrement des murs dans ce secteur, tant les terrasses modernes, les eboulementset les enclos en ont boulevers ce organisation. Un exemple de possible réappropriation de maison ancienne, ou tout au moins de remploi Antonios l'́d difice n'est pas reportésur le phan de J Demargne, mis les remploiser réoccupation ne font aucun doute. Les murs de la maison ont été en grande partie reconstruitsà laidede mater riaux prisà proximite, les fondations del ancien edifice son visibles a intererieur de la maison moderne qui sert aujourd hui c abri pour les bergers

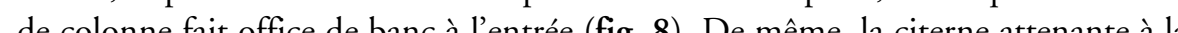
chapelle à l'Ouest est probablement une construction antique réaménagée, comme en témoigne la qualité de l'enduit hydraul lique observe à l'intérieur ainsi que les dimensions du regard, similaires à celles des citernes du site. Ces deux structures prouvent en

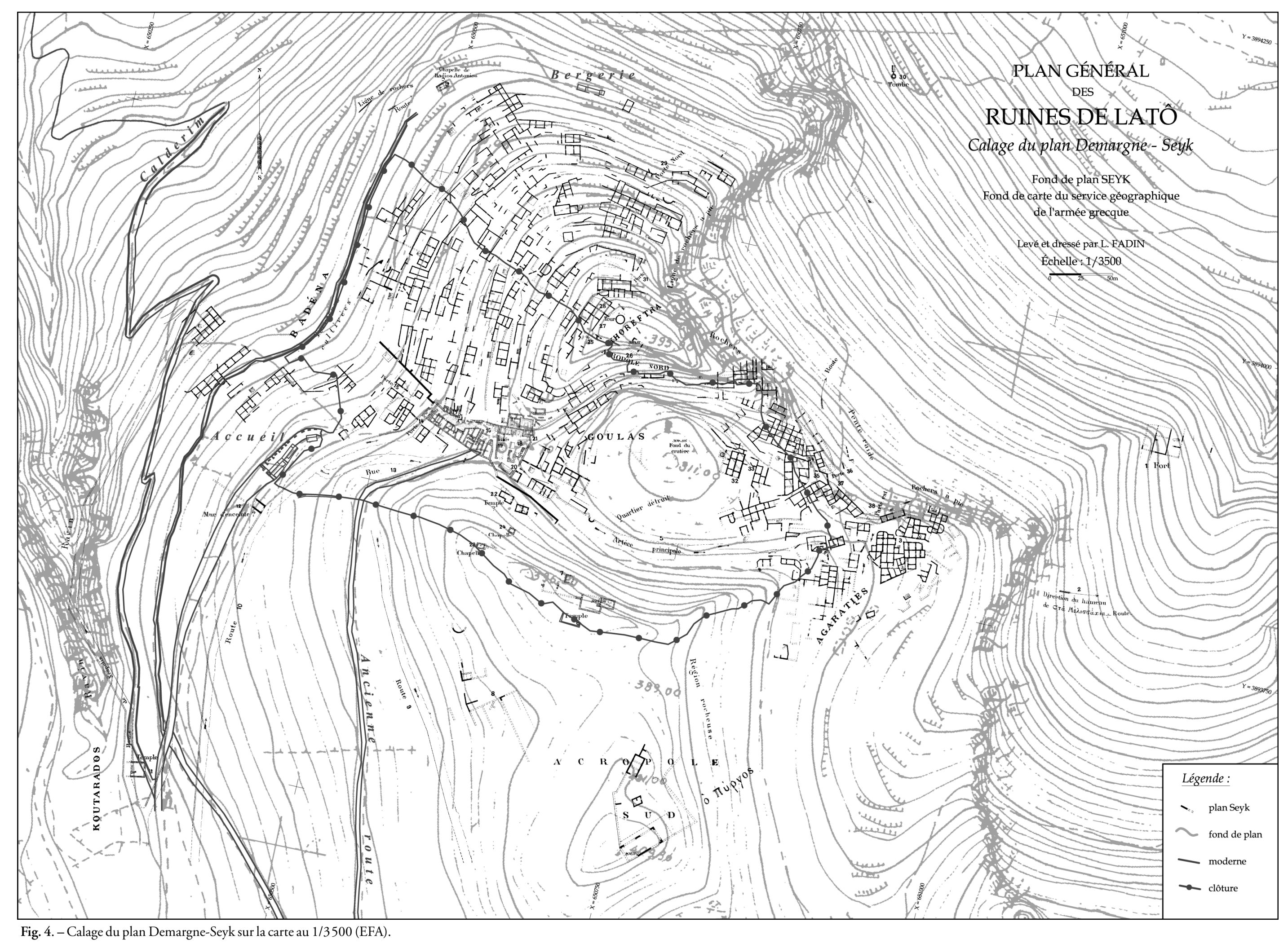


Les interférences que produisent la végétation et les différentes traces de réoccupation ne nous ont donc pas permis de retrouver les conditions d'observation qui étaient celles du début du siècle et ont laissé dans l'ombre des vestiges que J. Demargne avait pourtant identifiés. Dans un certain nombre de cas, le plan reste ainsi le seul témoignage de bâtiments éboulés, noyés dans la végétation ou masqués par des constructions modernes. De ce point de vue, les apports du plan de 1901 restent indéniables : dès cette époque les limites de la ville et des quartiers d'habitation ont été parfaitement évaluées.

\section{B) MESURE DE LA FIABILITÉ DU PLAN SEYK}

Pour mener à bien cette mesure, nous disposions dès le départ d'une documentation topographique importante. Si les cartes aux 1/50 000 et 1/100 000 ne sont pas disponibles à la vente auprès du $\Gamma \Upsilon \Sigma(\Gamma \varepsilon \omega \gamma \rho \alpha \phi i \kappa \eta ́ \Upsilon ~ \Upsilon \Uparrow \eta \rho \varepsilon \sigma i \alpha \Sigma \tau \rho \alpha \tau o v ́)$, nous avons pu acheter, dans un premier temps, les quatre cartes au 1/5000 qui permettent de couvrir la région immédiate de Latô. Elles ont été utilisées pour localiser et vérifier les trois bornes géodésiques qui servent au calage des relevés actuels. À partir des coordonnées des bornes portant les numéros 506 (au Sud-Ouest du site), 509 (à l'Ouest) et 436 (à l'Est) dont nous avons pu vérifier l'homogénéité, trois stations principales (clous d'arpentage enfichés et fixés avec de la colle époxy dans le rocher) et trois stations de contrôle (croix dessinées à la peinture rougeà proximité de chaque clou) ont été implantées de manière pérenne à Latô même, sur l'acropole Nord, sur l'acropole Sud et sur le pyrgos. Les six points ont tous été mesurés avec deux méthodes GPS différentes : en RTK ${ }^{45}$ avec une moyenne sur dix mesures successives et en statique rapide ${ }^{46}$ pendant 20 minutes. Enfin, nous avons stationné chacun des trois clous matérialisant les points de calage avec la station totale et mesuré les distances et les angles depuis ces trois points vers les autres points de référence et les deux bornes 506 et 509, obtenant ainsi un deuxième contrôle du positionnement des points d'appuis. Il était indispensable de connaître avec précision et certitude les coordonnées de ces points qui ont servi au rattachement du plan au système de projection grec EGSA $87^{47}$ et au géoréférencement des vestiges établi par V. Seyk.

45. R.T.K. (Real Time Kinematic) est une technique de positionnement par satellite utilisée par un GPS différentiel en temps réel. L'antenne mobile reçoit les données transmises par l'antenne fixe (référence ou pivot), les combine avec ses propres données et calcule les coordonnées en temps réel, permettant d'atteindre une précision de l'ordre du centimètre ; les coordonnées du pivot doivent être connues avec la meilleure précision possible.

46. Méthode de positionnement par GPS fondée sur la résolution des ambiguïtés sur de très courtes périodes d'observation. Cette technique nécessite un travail de calcul en post-traitement. Le récepteur de référence observe les satellites de façon continue tandis que le récepteur mobile visite les autres points.

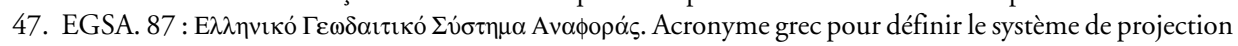
utilisé depuis 1987 pour la géodésie, la topographie et la cartographie en Grèce. 
Pour compléter l'analyse, des relevés de coupes sur le terrain ont été pratiqués afin, d'une part, de faire au relief la place qu'il mérite dans les documents graphiques accompagnant l'exploration d'un site escarpé comme Latô ; d'autre part, d'aider au « calage » des vestiges repérés par Seyk et, partant, de tout le plan. Une grande partie de la mission du mois d'août 2006 a été consacrée à l'établissement de deux coupes transversales Est-Ouest et Nord-Sud $(1010 \mathrm{~m})$ au 1/200. Elles donnent le profil général du site, à la fois dans ses caractéristiques géographiques (altitude, dissymétrie des versants, importance des pendages) et topographiques (implantation et répartition des différents quartiers, système des terrasses, lignes de défense naturelles). Elles sont réalisées au GPS ou au tachéomètre selon les caractéristiques du terrain et seront utilisées dans l'analyse de la morphologie urbaine de Latô dont nous annonçons la publication prochaine dans l'introduction. L'escarpement du site et la difficulté d'accès de certains secteurs nécessitaient de réfléchir aux points de passage et aux axes de coupe : la coupe Nord-Sud est ainsi levée en deux parties dont la deuxième s'infléchit légèrement vers l'Est à partir de la tour pour descendre dans la doline. Le relevé de ces coupes a souvent été rendu très difficile par deux obstacles majeurs : l'importance de l'à-pic de l'acropole Nord vers la doline et la végétation extrêmement dense des pentes de cette dernière ont rendu nécessaire le déboisement complet d'un large passage pour permettre la visée au tachéomètre (fig. 9). Les coupes localisées sont volontairement limitées au Quartier Ouest, afin de comprendre l'implantation, l'extension et l'orientation des terrasses dans une zone étendue et contrastée. L'élaboration de ces coupes permet en outre de circuler de part et d'autre de son axe et de reconnaître voire de relever d'autres structures caractéristiques, notamment des citernes inédites ou des bâtiments absents du plan Demargne. L'implantation est faite après un travail de repérage mené en confrontant les informations du plan et du terrain. Durant la mission, trois coupes localisées ont été levées dans le Quartier Ouest, donnant ainsi les principales orientations de la pente et soulignant les différences d'implantation des terrasses qui l'étagent. Comme pour les grandes coupes transversales, le relevé des points est systématiquement accompagné de prises de niveaux et de l'élaboration d'un croquis pour faciliter la mise au net.

À la demande de l'éphorie, l'ensemble des structures modernes qui entourent Latô a été relevé en même temps au GPS, afin de rendre compte de l'état complet et actuel du site : la clôture moderne, les deux bâtiments d'accueil, la bergerie du versant Nord, les deux routes menant au site (l'ancien accès ainsi que le nouveau), le chemin qui conduit à la chapelle d'Aghios Antonios et le caldérim, ont ainsi été géoréférencés, dessinés et intégrés au plan Demargne et à la carte au 1/5000 (fig. 4).

BCH 131 (2007) 


\section{C) ANALYSE DES DIFFICULTÉS RENCONTRÉES}

Les difficultés rencontrées pour le « calage » du plan Seyk ont été nombreuses. Elles peuvent s'analyser comme suit.

- Il faut ici rappeler que nous ne disposons, dans les archives de l'École française d'Athènes, d'aucun document original du plan, sous sa forme achevée ou partielle. Nous ignorons donc à quelle échelle il a été réalisé avant d'être gravé et publié au 1/2000 dans le Bulletin de Correspondance Hellénique de 1901. L'information serait d'importance puisqu'elle nous permettrait de mesurer l'ampleur des distorsions dues au changement d'échelle, à la gravure, au pliage du papier, à l'impression et à la reproduction. Quoi qu'il en soit, et après plusieurs essais de calage du plan, les différences observées entre le relevé de J. Demargne et les mesures effectuées sur le terrain sont de l'ordre d'une quinzaine de mètres et peuvent aller jusqu'à vingt mètres. L'orientation des distorsions est hétérogène. Les différences sont plus sensibles dans les parties les moins fiables du plan, aux extrémités et dans les zones où le semis des points de repère que nous avons choisis n'est pas homogène. Dans certains secteurs en effet, il a été difficile de repérer des points d'ancrage dont nous pouvions être sûrs qu'ils correspondaient aux structures dessinées sur le plan. Le traitement numérique du plan Seyk par le logiciel MapAnalyst a mis en évidence des problèmes non uniformes de distorsion, mais n'a pas offert de solution de recalage. La solution adoptée in fine ressemble à celle que l'on appliquait autrefois avec un simple calque : le plan a été mis à l'échelle de la carte au 1/5000 et centré sur un point certain, en l'occurrence, le centre de la tour ronde située au sommet de l'acropole Nord. Il a ensuite subi une rotation autour de ce point en direction de la chapelle d'Aghios Antonios (fig. 4).

- La charte graphique employée dans la réalisation du plan peut également être source d'erreur dans la reconnaissance des vestiges sur le terrain. Si certains édifices sont identifiés par J. Demargne à l'aide de numéros développés dans la légende, le plan et l'article ne comportent pas d'explicitation des graphismes utilisés. Un trait continu associéà un trait pointillé signale un mur de terrasse et les rues principales de la ville, mais leur signalement n'est pas systématique et le mur de soutènement peut aussi être un mur de terrasse $^{48}$. La partie comprise entre la Porte Ouest et la limite Est de l'agora ayant été

48. À partir des définitions données par R. GINOUVÈs, Dictionnaire méthodique de l'architecture grecque et romaine, vol. III (1992), p. 21, on peut déduire que le mur de terrasse contient un espace libre de construction, tandis que le mur de soutènement détermine un espace construit. Dans le cas de Latô, la différence entre le mur de terrasse et le mur de soutènement n'est pas systématique. Une terrasse peut en effet recevoir un ou plusieurs édifices. Par ailleurs, la largeur limitée des espaces disponibles entre deux courbes de niveau nécessite souvent que le mur de terrasse soit aussi un mur de soutènement. Pour les mêmes raisons, le mur de soutènement se prolonge parfois en hauteur pour constituer le mur de façade de l'édifice luimême. 


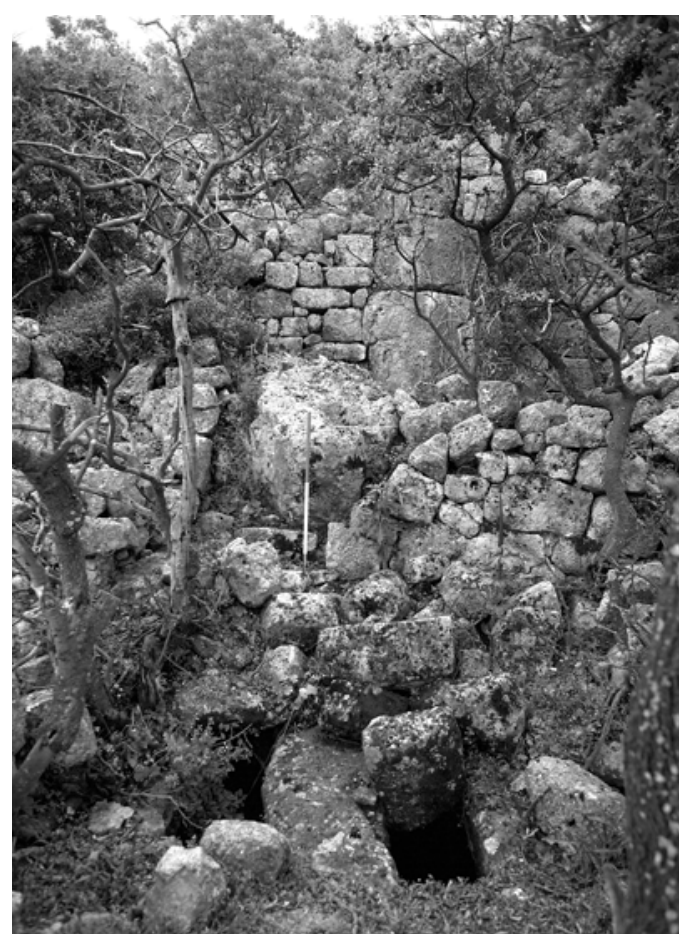

Fig. 10. - Exemple de citerne découverte dans le quartier Nord (cliché H. Wurmser, EFA).

fouillée et relevée, nous disposons de détails et d'une précision dont les autres secteurs de Latô n’ont pas nécessairement bénéficié49

- La nouvelle exploration du site a permis de localiser un certain nombre de constructions qui n'avaient pas été relevées par J. Demargne. De nombreuses citernes inédites ont ainsi été repérées et géoréférencés à l'aide du GPS (fig. 10). Leur absence sur le plan peut facilement s' expliquer par le fait qu' elles sont le plus souvent éboulées ou masquées par la végétation. La présence de certaines citernes a été déduite grâce à une attention particulière portée à divers signes : la présence de lichens et de mousse attestant une humidité résiduelle, la forme en cuvette de l'éboulis, les traces d'enduit hydraulique, la proximité d'une maison, d'une rue ou d'un mur de terrasse. Le fait qu'aucune fouille exhaustive n'ait apparemment été menée au sein des quartiers d'habitation, a probablement conduit l'architecte Seyk à ne signaler que les citernes dont une partie au moins

49. Voir bibliographie donnée n. 43. 


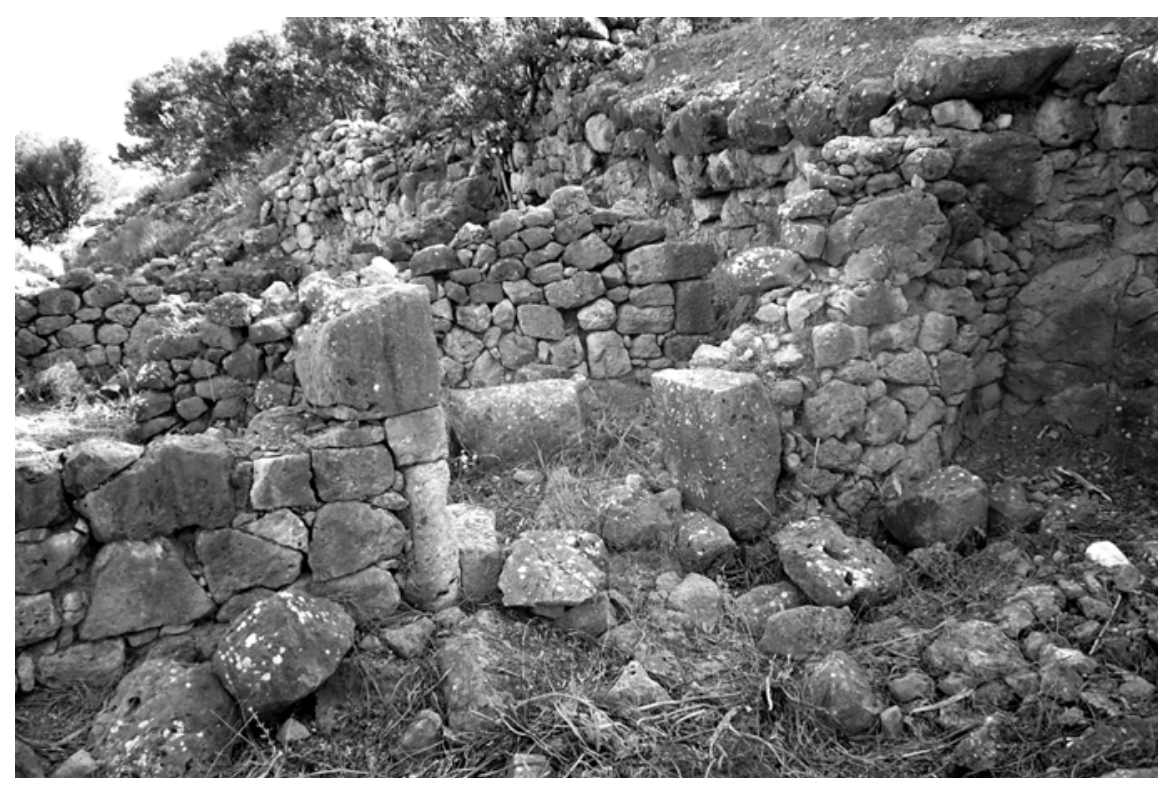

Fig. 11. - Maison du Quartier Ouest ne figurant pas sur le plan de 1901 (cliché H. Wurmser, EFA).

de la couverture et de la forme générale était visible. Des citernes ont ainsi été identifiées avec certitude au cours des missions 2005-2007 dans les quartiers Ouest et Nord, notamment lors des explorations préparatoires au tracé des coupes localisées et lors du déboisement de certains secteurs pour permettre la visée au tachéomètre. On ajoutera à celles-ci la citerne découverte lors de l'étude d'une maison dans le secteur d'Agaratiès par V. Hadjimichali ${ }^{50}$. Elle constitue en effet l'un des rares exemples de citerne entièrement conservée du fait de son enfouissement total jusqu'au moment de la fouille.

- Le plan Demargne doit aussi être complété par le relevé de structures visibles aujourd'hui grâce aux fouilles menées à la fin des années soixante par O. Picard, P. Ducrey et V. Hadjimichali. L'exèdre Est et le théâtre étaient en effet enfouis sous les déblais et les éboulis au moment du relevé du plan complet. Au sein du Quartier Ouest en revanche, un édifice entier semble avoir été oublié par l'architecte V. Seyk, alors qu'il est aujourd'hui parfaitement visible et dégagé (fig. 11). Aucune fouille dans ce secteur n'a pourtant été effectuée dans le cadre des missions Picard - Ducrey - Hadjimichali. L'édifice dont il s'agit est très probablement une maison, similaire à celles qui ont été étudiées par 
V. Hadjimichali51. Elle est constituée de quatre pièces en enfilade et pourvue d'un accès à une citerne située légèrement en contrebas et au Sud. La citerne figure bien sur le plan, mais pas la maison. La présence de ce bâtiment, ainsi que la difficulté à reconnaître qu'il pût s'agir d'un oubli, nous a conduits dans un premier temps à interpréter fautivement l'organisation du secteur et les rapports qui unissent la citerne et les bâtiments environnants. Lors d'une opération de nettoyage, la découverte d'une seconde citerne importante, construite à une quarantaine de mètres au Nord-Est, a permis de caler l'ensemble et de conclureà l'absence de la maison sur le plan Demargne. Cette absence nous laisse cependant d'autant plus perplexes que l'édifice semble avoir été fouillé. Le mur Est de la maison, qui est aussi un mur de soutènement, est en effet conservé sur plus de deux mètres de hauteur ; les seuils et piédroits sont en place et correspondent manifestement au niveau d'occupation ; aucun éboulis n’a perturbé le plan général et aucune végétation d'importance n'a poussé à l'intérieur des pièces ; un cône de déblais anciens a pu être repéré à proximité de la citerne Sud. Il semble donc probable que la maison était presque totalement invisible à la fin du XIXe siècle, au moment où J. Demargne et V. Seyk effectuaient le relevé complet du site : seuls quelques tronçons de murs paraissent avoir été dessinés, notamment à l'Est où la présence de lichens sur le haut des dernières assises montre que le mur affleure à l'air libre depuis l'enfouissement du bâtiment. La consultation des archives manuscrites de l'École française d'Athènes a permis de constater que la maison apparaissait dans le dossier photographique élaboré par V. Hadjimichali pour l'étude de l'habitat latien, sans pour autant qu'elle ait utilisé cette documentation dans la publication finale. Il faut donc conclure que la maison a été fouillée entre le début du XXe siècle et les campagnes des années soixante. Il est très peu probable, en revanche, qu'il s'agisse d'une réoccupation moderne imputable à la présence régulière de bergers et d'habitants de Kritsa venus récolter les fruits des amandiers et des oliviers : les réoccupations sont en effet davantage concentrées autour de l'agora de Latô et n'ont dû en aucun cas nécessiter des travaux de dégagement aussi importants. Si l'on admet que seul le mur Est de la maison était partiellement visible, il n'était pas non plus possible de savoir à l'avance quel type de construction était enfoui et quel usage pouvait en être fait. Nous avons donc tendance à attribuer le dégagement de ce bâtiment aux opérations effectuées par A. Reinach à la suite de J. Demargne, opérations que nous ne connaissons qu'indirectement et qui n'ont jamais été localisées par leur auteur.

- Mais le repérage de nouvelles constructions ne va pas sans poser des questions chronologiques parfois difficiles à trancher : s'agit-il à chaque fois de constructions véritablement antiques ? Un mur de pierres sèches n'étant pas en soi caractéristique d'une 
période, seule la fouille pourrait éventuellement, mais sans garantie, apporter des éléments de datation. Par ailleurs, le maintien à Latô de traditions constructives fortes invite à interpréter avec prudence l'évolution des appareils de murs et à utiliser avec circonspection les typologies traditionnelles de la construction. De la même façon, les murs de terrasse antiques ne sont pas toujours différenciables de leurs homologues modernes. L'environnement immédiat du site est en effet régulièrement aménagé en terrasses de culture, notamment à l'Est et il est probable que ce type d'adaptation aux conditions naturelles du terrain était déjà en usage dans l'Antiquité. Il est ainsi difficile d'interpréter l'absence sur le plan du dispositif présent dans le fond de la doline. Il s'agit d'un mur épais adoptant une forme circulaire incomplète, fait de blocs de pierre massifs ou partiellement taillé dans le rocher ; aucune trace de ce mur n'apparaît cependant dans le plan de 1901. Il pourrait constituer une sorte de point de captation des eaux de ruissellement provenant de l'acropole Nord, hypothèse accréditée par la présence d'une couverture végétale dense à cet endroit et constituée d'essences d'arbres ayant particulièrement besoin d'eau, mais il est pour l'instant impossible d'en établir la date.

- L'étude menée sur l'habitat de Latô durant les campagnes plus récentes de la fin des années soixante permet également de réviser le plan de certaines maisons dont le tracé était parfois schématique ou fautif ${ }^{52}$. Dans un certain nombre de cas, le plan a fourni une localisation précise et exacte des bâtiments, mais leur dessin s'est révélé faux ou très incomplet. La plupart du temps, quand la végétation et les éboulis n'étaient pas trop importants, les murs extérieurs des constructions ont été identifiés sans difficulté particulière. En revanche, les séparations intérieures notées sur le plan ont été difficilement repérées sur le terrain. Certaines zones sont ainsi restées illisibles, notamment lorsque l'agencement des murs, déjà curieux sur le plan, ne pouvait être retrouvé dans les vestiges observables du fait de leur intrication. C'est notamment le cas de la partie basse des quartiers Ouest et Nord et du quartier situé sur la pente Ouest de la doline. De ce point de vue, les fouilles réalisées par O. Picard, P. Ducrey et V. Hadjimichali ont contribué à rectifier la schématisation du plan d'origine en révélant des plans moins orthogonaux qu'il n'y paraissait. Contrairement à ce que suggère le plan de 1901, la Maison du Prytanée est constituée en réalité de deux maisons mitoyennes, légèrement décalées l'une par rapport à l'autre et possédant chacune leur entrée et leur citerne propres (fig. 12). D'autres rectifications de ce type ont été faites grâce à l'étude architecturale effectuée par V. Hadjimichali sur les maisons. Ainsi, pour la maison du Temple, la fouille a permis de dégager l'organisation intérieure de la construction et de

52. À titre d'exemple, les maisons-tours construites dans le mur fortifié de la rue Ouest, la Maison du Prytanée ; la Maison du Temple est en revanche une fouille entièrement nouvelle. La synthèse sur l'habitat a été effectuée par V. HADJIMICHALI (supra, n. 44), p. 167-222. 


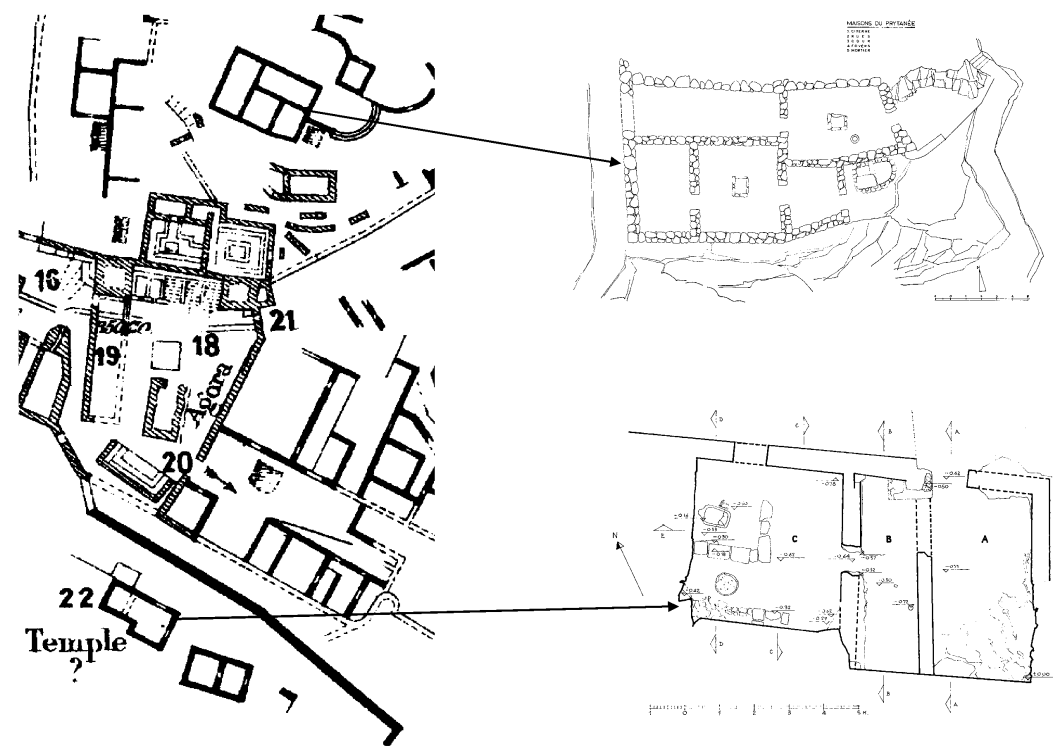

Fig. 12. - Maison du Quartier Ouest ne figurant pas sur le plan de 1901. Comparaison des plans réalisés par J. Demarge et V. Hadjimichali.

compléter les informations sommaires fournies par le plan Demargne, sur lequel ces vestiges sont, par erreur, identifiés avec le temple lui-même.

Les problèmes posés par le plan Demargne aujourd'hui sont donc nombreux. L'analyse minutieuse des écarts entre l'observation de terrain et le dessin nous a cependant permis de mieux cerner les méthodes utilisées par J. Demargne et V. Seyk : par leur schématisation et leur complexité, il apparaît que certaines zones ont probablement fait l'objet d'un levé de points reliés ultérieurement, loin du site, à partir de croquis pris sur place. Le document reste toutefois une base de travail d'autant plus indispensable que nous en connaissons aujourd'hui les limites et les défauts. Selon les cas, des relevés de détail, des prises de points et de courbes de niveau, ainsi que le repérage systématique de nouvelles structures, édifices ou citernes, permettent de compléter le plan et d'améliorer sa lisibilité. Les missions effectuées entre 2005 et 2007 nous ont au demeurant montré à quel point la réédition d'un tel plan serait encore aujourd'hui un travail gigantesque. Outre la dégradation régulière des conditions d'observation des vestiges, les exigences financières, humaines et techniques d'une telle entreprise nous semblent à tout le moins démesurées, malgré les moyens modernes dont nous disposons. 


\section{TOPOGRAPHIE ET GESTION DU SITE (S. A., A. F., H. W.)}

La mise au point d'une documentation topographique solide permet d'accompagner et de préparer les aménagements du site rendus nécessaires par la fréquentation croissante des ruines à la belle saison. Ces travaux ont été entrepris depuis plusieurs années par la XXIV éphorie des Antiquités préhistoriques et classiques sous la direction de Mme $S$. Apostolakou. L'occasion est offerte aujourd'hui d'en faire une présentation synthétique.

En 1999, grâce à l'intérêt personnel d'A. Zervos, alors maire d'Aghios Nikolaos, et avec un financement de la municipalité, a été préparée une étude visant à la protection et à la mise en valeur générale du site archéologique de Latô. L'étude prévoyait conjointement la construction de bâtiments d'accueil des visiteurs ; l'aménagement de l'environnement des nouvelles constructions et de l'accès au site archéologique ; la démolition de l'ancien grillage et l'implantation d'une nouvelle clôture ; la restauration du site archéologique ; l'élaboration et la mise en place de panneaux d'information. L'étude fut approuvée par le Ministère de la Culture, inscrite au Programme d'Action Régionale de Crète et financée par le deuxième Plaisio municipal de soutien. La réalisation du chantier en 2000 fut confiée à l'Organisme de Développement de la Crète de l'Est qui a partagé avec la XXIVe éphorie des Antiquités préhistoriques et classiques la surveillance des travaux.

Pour la construction des bâtiments d'accueil, la municipalité d'Aghios Nikolaos a cédé une surface de $1000 \mathrm{~m}^{2}$ au Sud de la zone archéologique, à l'extrémité de la route qui mène de Kritsa à Latô. L'existence d'un cheminement déjà aménagé pour les visiteurs fut un critère déterminant pour le choix de cet emplacement qui permettait en outre de dissimuler les constructions modernes depuis le site. Trois bâtiments distincts - vente de billets, cafétéria, toilettes - d'un seul niveau, de petites dimensions et de formes simples ont été installés dans l'espace disponible. Dans l'intention de les intégrer au mieux dans l'environnement en les faisant ressembler aux anciennes maisons crétoises des hameaux de la région, on a utilisé le bois et la pierre, notamment et principalement des pierres provenant des déblais de fouilles répartis en différents endroits du site. Ce mode de construction permet à la fois d'obtenir un effet «d'ancien » et de résoudre, au moins partiellement, le problème récurrent de l'évacuation des déblais de fouilles accumulés. Lors du tri des pierres on s'est efforcé de ne pas employer celles qui pourraient un jour être utilisées dans les travaux de consolidation ou de restauration des monuments. Des dalles, des banquettes de pierre, des plantations d'arbustes ou de végétaux de la flore locale en jardinières complètent les aménagements de l'entrée et de l'accueil. Pour le bon fonctionnement des bâtiments, ainsi que pour assurer la protection du site contre l'incendie, le service archéologique a installé des réservoirs et un réseau d'acheminement de l'eau sur une distance de 1200 mètres ; les installations bénéficient en outre d'une alimentation électrique assurée par le réseau de Lakonia.

Afin de protéger le site contre le bétail errant, l’ancienne clôture qui enserrait les 85120 $\mathrm{m}^{2}$ de terrains expropriés a été remplacée par un nouveau dispositif à structure renforcée 
d'une longueur totale de $1,850 \mathrm{~km}$ et d'une hauteur de 2 mètres. La clôture est constituée de tubes en acier galvanisé, particulièrement efficaces pour la protection du site, et d'un grillage plastifié de couleur verte qui s'intègre au mieux dans l'environnement. Un relevé complet de celle-ci a été réalisé en 2006 par l'École française d'Athènes (fig. 4).

Pour faciliter et sécuriser la circulation des visiteurs, le sentier qui conduit à la porte Ouest de la ville a été amélioré. Un dallage et des degrés ont été aménagés dans le rocher aux points de passage difficiles, tandis qu'a été rétabli le mur de soutènement en pierres sèches aux endroits où il s'était effondré. Des travaux étendus de nettoyage et de déboisement ont été effectués dans la plus grande partie de l'espace ouvert aux visiteurs afin de faciliter les travaux de consolidation et de réfection des monuments. L'ancienne balustrade détériorée qui entourait la citerne centrale de l'agora a été remplacée par un garde-corps en métal de couleur verte. À l'entrée du site, le service archéologique a placé un grand panneau représentant le territoire des Latiens au II $s$. av. J.-C., tel que le révèle l'étude des témoignages épigraphiques, tandis que sur l'agora, un second panneau présente un plan des bâtiments publics de la cité. Des panneaux de plus petite taille ont été également réalisés pour indiquer en trois langues le nom des bâtiments isolés.

Dès l'exploration archéologique de J. Demargne et jusque dans les années 60, le site a subi de nombreuses altérations dont il a été question dans les précédents chapitres de cet article. Les monuments ont en outre particulièrement souffert de la fréquentation non contrôlée des visiteurs et de la continuelle circulation des troupeaux de chèvres et de moutons dont le piétinement entraîne régulièrement la chute des murs anciens. Des travaux restreints de consolidation et de sauvegarde ont de ce fait été entrepris en 1978 et 1994 . Une campagne plus systématique de restauration des murs a en outre été conduite dans les monuments déjà fouillés et publiés. Cette entreprise a été effectuée avec le financement général des travaux de protection et de mise en valeur du site, sous la surveillance du restaurateur chevronné des antiquités de la XXIV éphorie des Antiquités préhistoriques et classiques, Alexandre Nikakis. Les travaux se sont concentrés dans les secteurs dela rue principale, dans les maisons qui s'étendent de part et d'autre de la rue Ouest, dans le sanctuaire hypèthre de l'agora, dans l'ensemble des pièces du Prytanée, dans les Maisons du Prytanée, ainsi que dans le temple. Pour protéger les monuments, un déboisement et un dessouchage de grande ampleur ont été effectués. Grâce à la contribution d'autres organismes, deux interventions sont aujourd'hui programmées dans l'environnement immédiat de Latô :

- La première concerne la viabilité du chemin agricole qui mène de la route locale KritsaLakonia à l'agora de la cité antique. L'objectif est de faciliter l'accès des personnes à mobilité réduite qui ne peuvent emprunter le cheminement existant, en leur offrant un espace d'où ils pourraient découvrir le quartier central de la ville avec l'aide d'un guide ou d'un panneau d'information.

- La seconde concerne un chantier de nettoyage et de restauration du caldérim sinueux qui monte au massif à partir de la plaine de Lakonia et constitue une solution alterna- 
tive pour tous ceux qui souhaitent combiner à la visite du site une randonnée pédestre. En 2004, l'administration des Eaux et Forêts du Lassithi a réalisé une étude d'aménagement, de mise en valeur et de protection des zones de beauté naturelle, des sites remarquables, ainsi que des lieux et régions répondant au label NATURA. Dans ce chantier en cours de réalisation ont été inscrits l'aménagement de la voirie et la signalisation du chemin dallé depuis Flamouriana jusqu'à Latô. Cette route dallée, longue de 2,5 km environ, représente le tronçon le mieux conservé de la voie qui, jusqu'au début du XXe $s$. reliait Kritsa à Iraklio et à Néapolis, l'ancienne capitale du Nome du Lassithi. La voie est d'ailleurs identifiée avec vraisemblance à l'ancienne route qui menait les Latiens aux terres qu'ils cultivaient dans la plaine de Lakonia et à Kamara. Il s'agit sans nul doute du chemin emprunté par Spratt lors de sa visite à Latô à partir d'Aghios Nikolaos. Dans un article de la Gazette Générale de Grèce ${ }^{53}$, on rapporte qu'une embuscade a eu lieu entre des Grecs et des Turcs qui empruntaient ce chemin lors de leur marche les ramenant de Sitia à Néapolis via Kritsa. La voie est également mentionnée par V. Psillakis ${ }^{54}$ : « Le 17 novembre 1867 un soldat Turc venant de Iérapétra et allant vers le Nord suivit la route qui conduit à Néapolis du Mirambello en passant par Meseleron, Kritsa et Lakonia ». Dans un article du journal Iraklio daté du 21 novembre 1894, à propos du chantier gigantesque d'aménagement des voies et des ponts dans le secteur du Mirambello, la route est mentionnée comme "la plus centrale et la plus longue voie de Malia à Kavousi ». Litinéraire est enfin signalé dans le journal Mesogios du 23 juillet 1895, dans un article intitulé "Carnets de route " : un voyageur et écrivain anonyme rapporte que, lors de son voyage de Néapolis à Sitia via Kritsa, il a passé la nuit dans une auberge "sise sur la voie publique et éloignée d'environ $2 \mathrm{~km}$ du village ». Cette route est restée en usage jusqu'au début du XXe siècle: en 1915, le conseil municipal de Kritsa a votéla réparation de la section allant d'Aghios Antonios à Margeli et de Firfiri Hani (qui se trouvait à mi-chemin environ de Latô et Kritsa) à Meskinian, Skafin, Xeropotamon, Koukistres, Pergiolikia jusqu’à Katharo55. Une étude visant à la préservation de cette route est en cours de réalisation.

Enfin, pour assurer la protection du site et la réalisation complète du programme de mise en valeur, l'éphorie prévoit d'une part, de continuer les travaux de consolidation des monuments; d'autre part, de poursuivreles expropriations des terrains de la ville antique en direction du Nord et de l'Est, sur une surface équivalente ou probablement plus importante que lors de l'expropriation de 1984, de manière à englober toute l'étendue des vestiges encore

53. "À propos des affrontements à Iraklio et à Sitia ", Gazette Générale de Grèce 31-32 (1829).

54. Histoire de Crète, IV , p. 323.

55. Les informations et les citations d'auteurs et de journaux sont dues à l'obligeance de Mme Maria Sorou, historienne. 
visibles dans la zone archéologique accessible aux visiteurs; leur protection sera ainsi assurée contre les destructions causées par les cultures et l'élevage du bétail et les travaux de restauration du site en seront facilités.

Au terme de l'évaluation des potentialités et des défauts du plan établi par J. Demargne et V. Seyk, il faut sans conteste rappeler que ce document reste fondamental pour les recherches actuelles et pour la mise en valeur du site de Latô. La vérification des repères géodésiques, l'ajout des courbes de niveaux, les compléments et les corrections apportés par les campagnes récentes, ainsi que le calage, même imparfait, du plan sur la carte au 1/5000 en font aujourd'hui un relevé largement utilisable pour de nouvelles études, à l'instar de l'analyse de la morphologie urbaine dont il sera question dans un prochain article. 